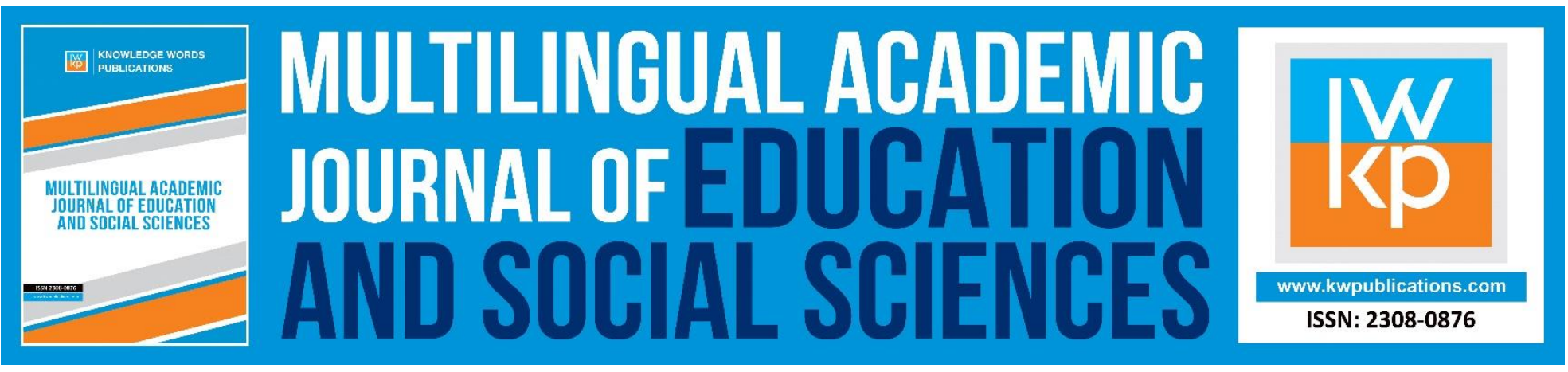

\title{
An Evaluative Approach of E-Learning Using Moodle in a Distance Post-graduate Course: A Case Study
}

\section{Georgios Papaioannou \& Eugenia A. Panitsides}

To Link this Article: http://dx.doi.org/10.46886/MAJESS/v8-i1/6969

DOI: 10.46886/MAJESS/v8-i1/6969

Received: 07 March 2020, Revised: 29 April 2020, Accepted: 04 May 2020

Published Online: 17 June 2020

In-Text Citation: (Papaioannou \& Panitsides, 2020)

To Cite this Article: Papaioannou, G., \& Panitsides, E. A. (2020). An Evaluative Approach of E-Learning Using Moodle in a Distance Post-graduate Course: A Case Study. Multilingual Academic Journal of Education and Social Sciences, 8(1), 52-65 (In Greek).

\section{Copyright: (C) The Authors 2020}

Published by Knowledge Words Publications (www.kwpublications.com)

This article is published under the Creative Commons Attribution (CC BY 4.0) license. Anyone may reproduce, distribute, translate and create derivative works of this article (for both commercial and non-commercial purposes), subject to full attribution to the original publication and authors. The full terms of this license may be seen at: http://creativecommons.org/licences/by/4.0/legalcode

Vol. 8, No. 1, 2020, Pg. 52 - 65

Full Terms \& Conditions of access and use can be found at https://kwpublications.com/pages/detail/publication-ethics 


\title{
An Evaluative Approach of E-Learning Using Moodle in a Distance Post-graduate Course: A Case Study
}

\section{Georgios Papaioannou \& Eugenia A. Panitsides}

Frederick University, Cyprus

\begin{abstract}
E-learning systems have significantly advanced online education, offering novel possibilities in distance education, such as attending solely online educational programmes. On these grounds, the purpose of the present study has been to investigate students' views on online courses and Moodle, taking into account easiness of use and communication possibilities both with professors and fellow students. To conduct the study, a qualitative approach was followed through the use of semi-structured interviews. The sample consisted of ten post-graduate students who attended the MEd. in Adult Education at Frederick university, Cyprus. The results demonstrated that most students have a positive view about distance online education, despite the fact that the opportunities offered for communication were rather limited. As far as Moodle is concerned, they are generally satisfied, considering it easy to use, and especially helpful in downloading educational materials. It was suggested among others that short-term training on ICT use should be provided to those students who wish to attend online post-graduate courses, so that lack of computer knowledge will not constitute a significant barrier.
\end{abstract}

Keywords: Adult Education, E-learning, Distance Education, LMS, Moodle, Assessment.

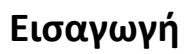

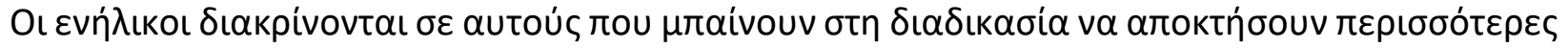

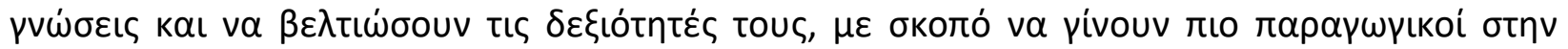

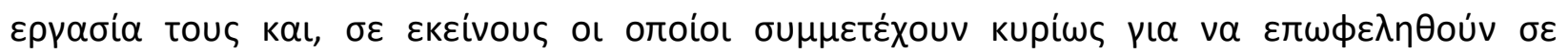

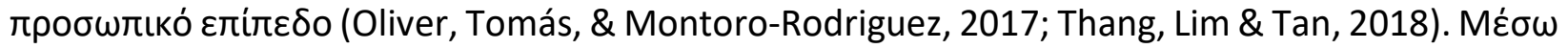

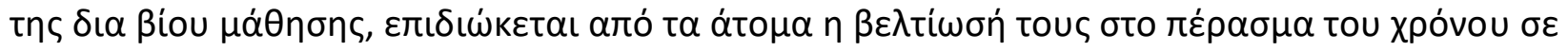

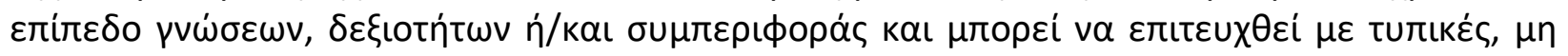

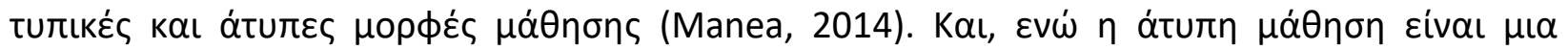

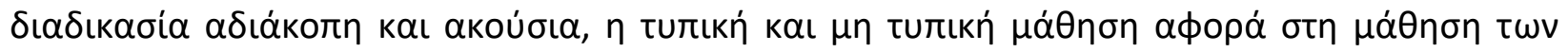

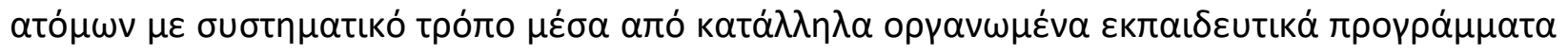
(Tudor, 2013; Khalid, Islam \& Ahmed, 2019; Alzgool, 2019). 
MULTILINGUAL ACADEMIC JOURNAL OF EDUCATION AND SOCIAL SCIENCES

Vol. 8 No. 1, 2020, E-ISSN: 2308-0876 @ 2020 KWP

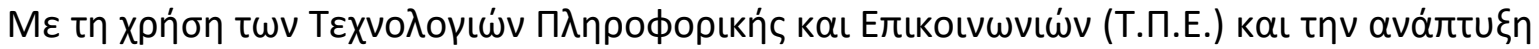

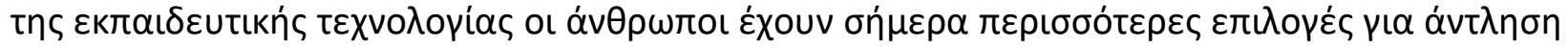

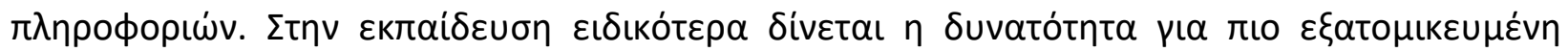

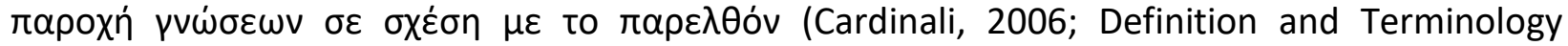
Committee of the Association for Educational Communications and Technology, 2008, or. $\alpha v \alpha \phi$.

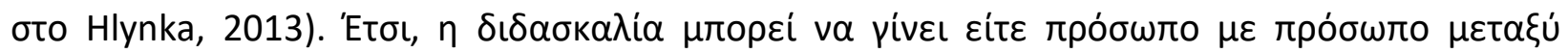

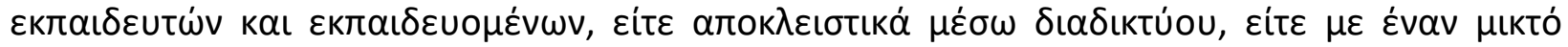

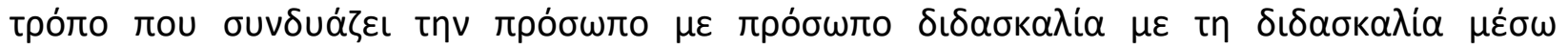

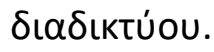

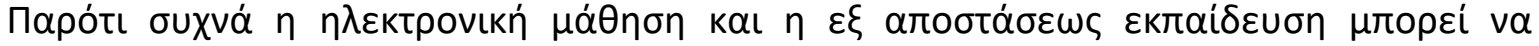

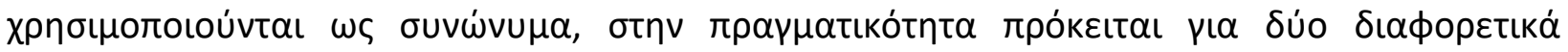

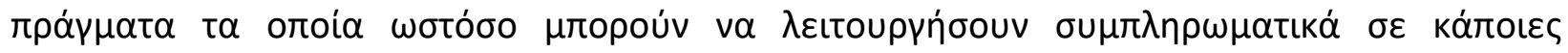

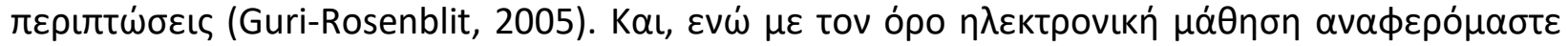

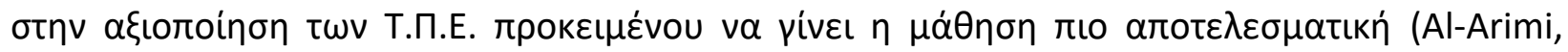

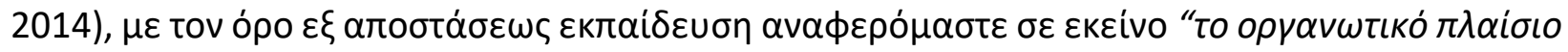

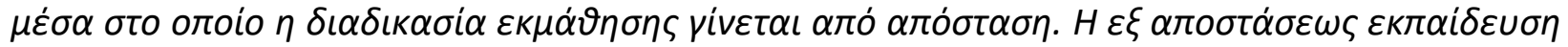

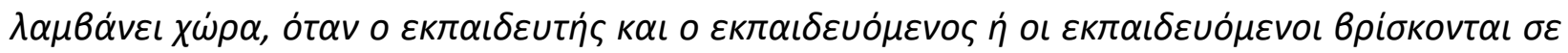

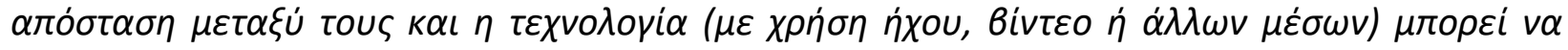

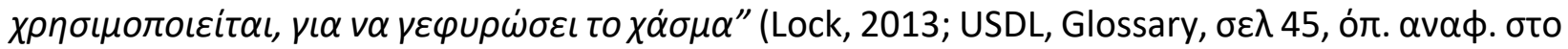
Kutluk \& Gulmez, 2012).

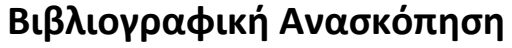

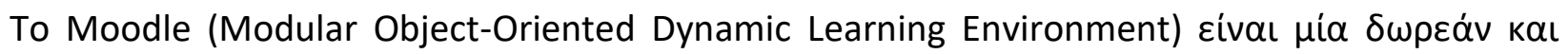

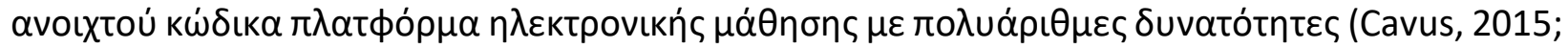

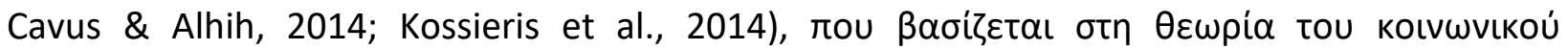

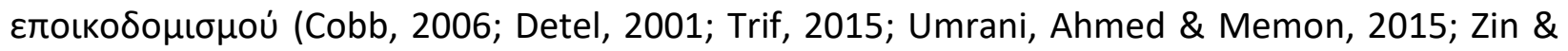

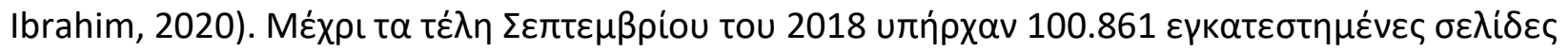

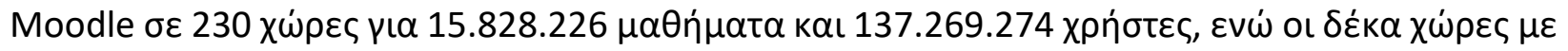

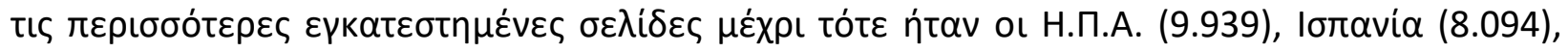

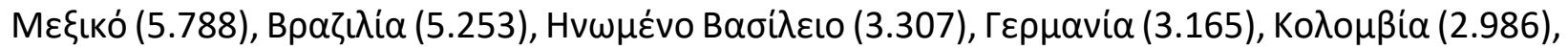

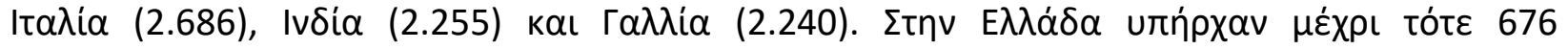

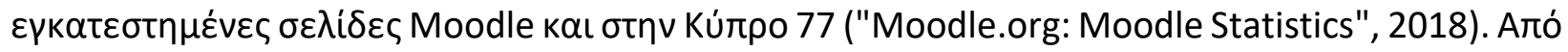

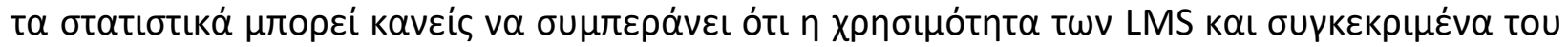

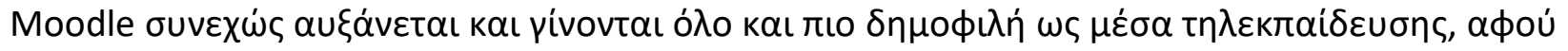

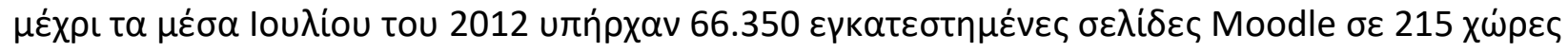

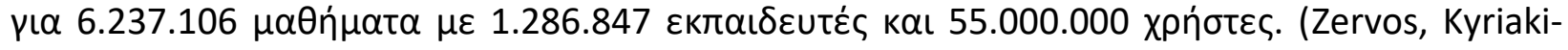
Manessi, Koulouris, Giannakopoulos \& Kouis, 2013).



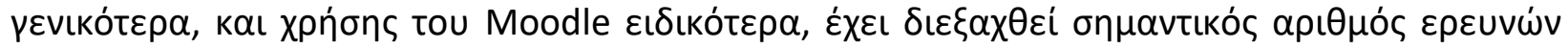

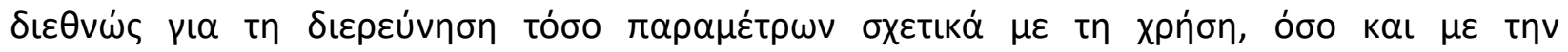

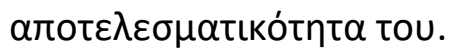

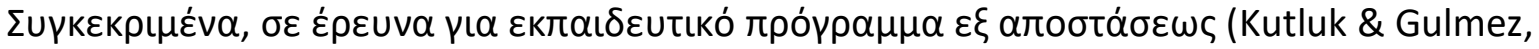

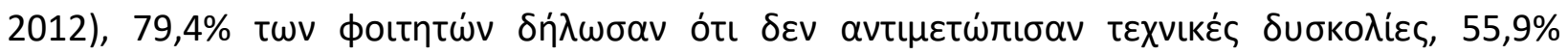

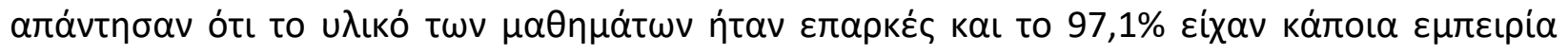


MULTILINGUAL ACADEMIC JOURNAL OF EDUCATION AND SOCIAL SCIENCES

Vol. 8 No. 1, 2020, E-ISSN: 2308-0876 @ 2020 KWP

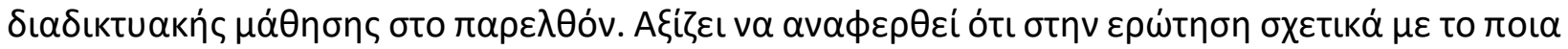

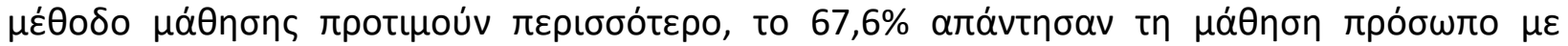

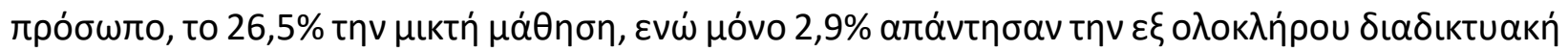

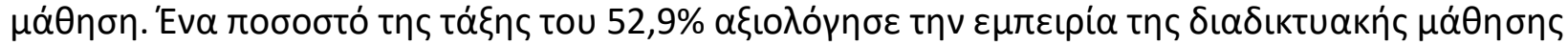

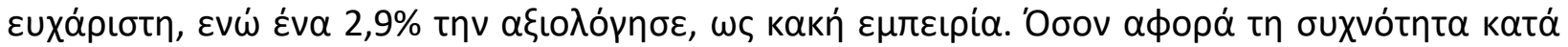

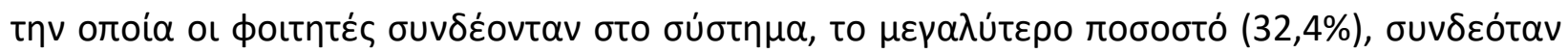

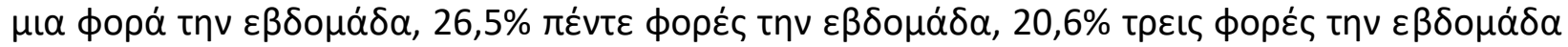

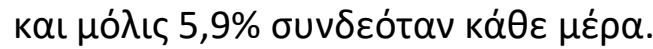

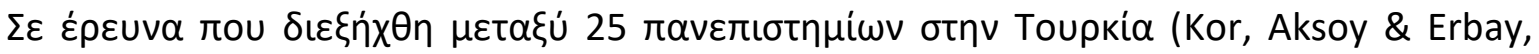

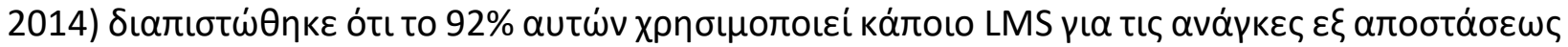

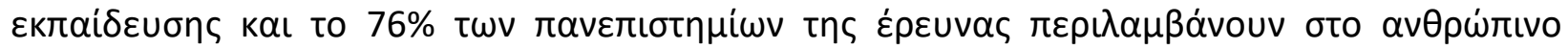

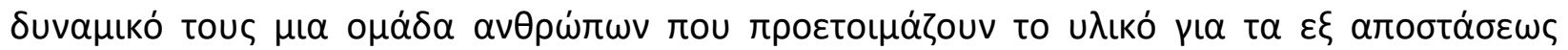

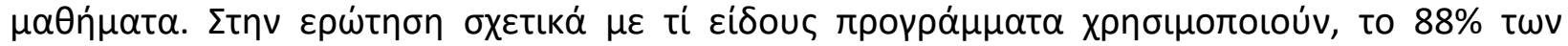

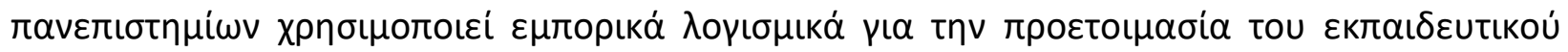

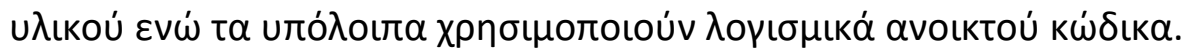

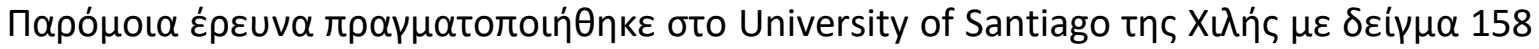

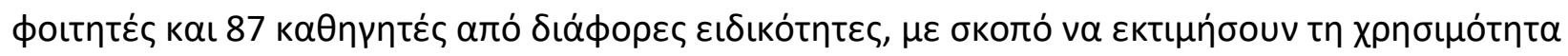

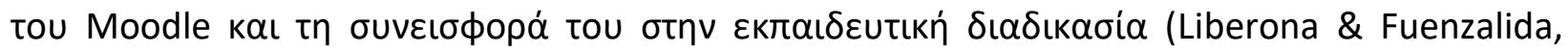

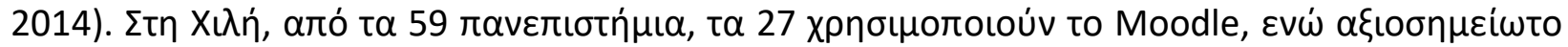

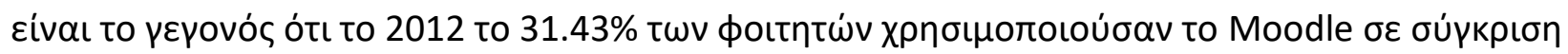

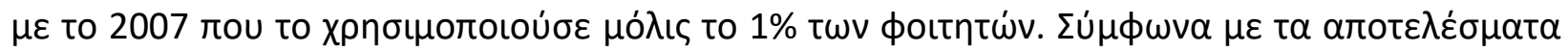

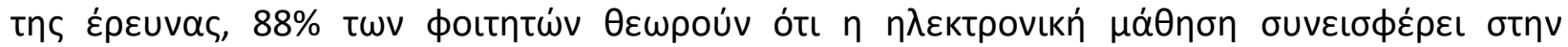
$\varepsilon \kappa \pi \alpha \iota \delta \varepsilon \cup \tau$ เ

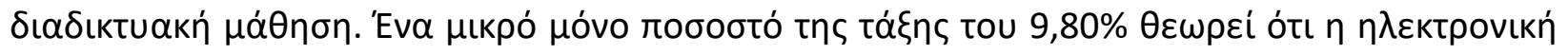

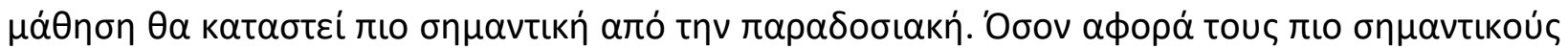

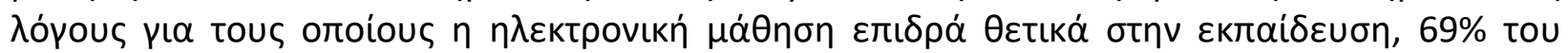

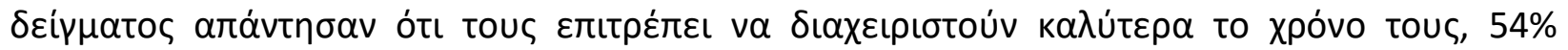

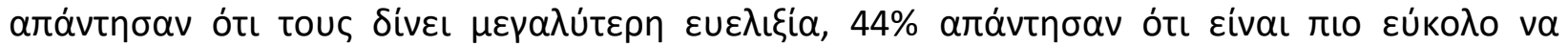

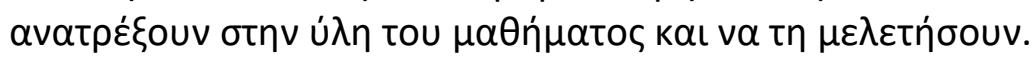

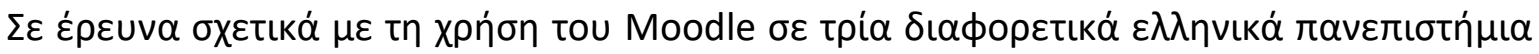

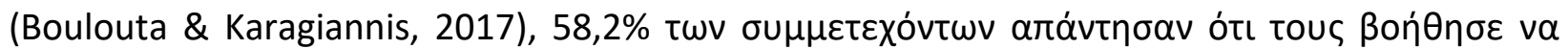

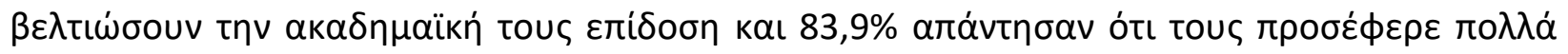

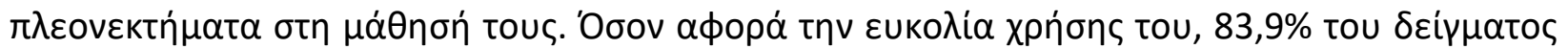

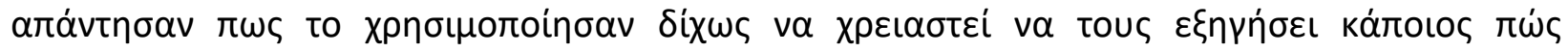

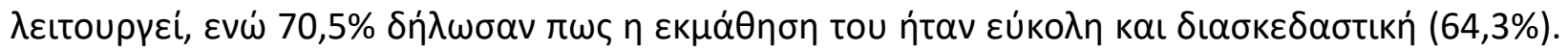

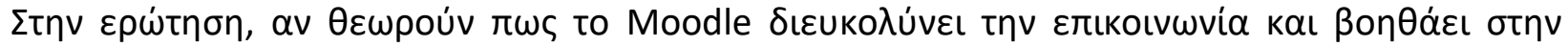

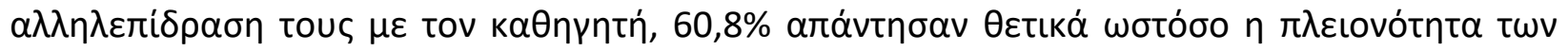

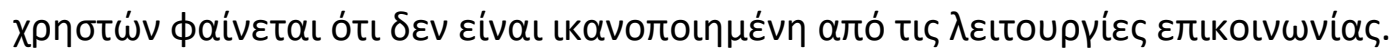

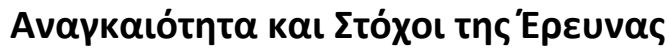

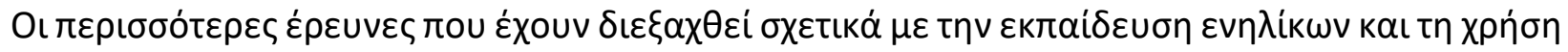

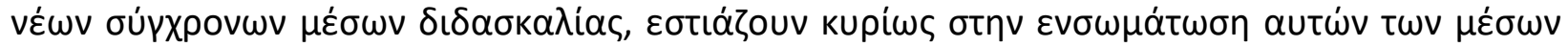

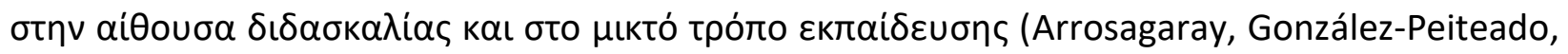
Pino-Juste \& Rodríguez-López, 2019; Deschacht \& Goeman, 2015). 
MULTILINGUAL ACADEMIC JOURNAL OF EDUCATION AND SOCIAL SCIENCES

Vol. 8 No. 1, 2020, E-ISSN: 2308-0876 @ 2020 KWP

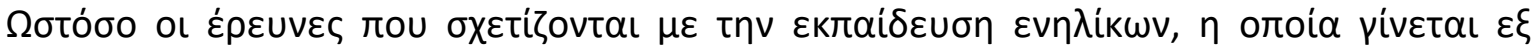

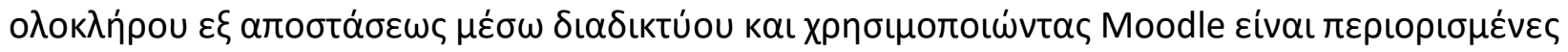

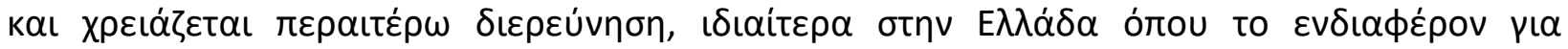

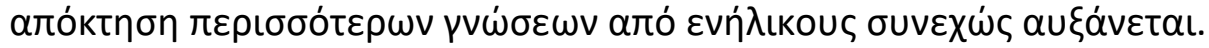

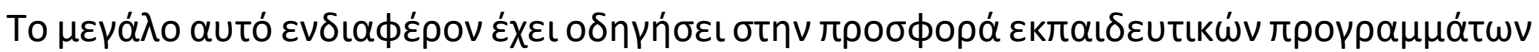

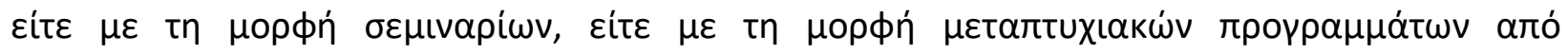

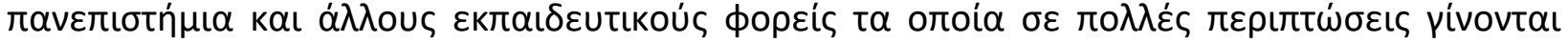

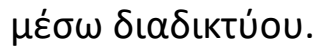

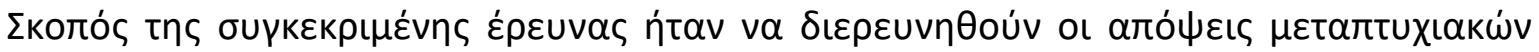

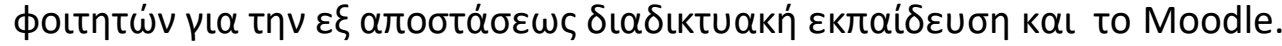

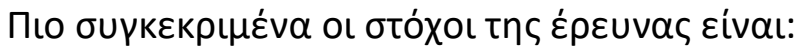

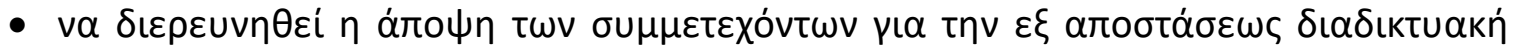
$\varepsilon \kappa \pi \alpha i \delta \varepsilon \cup \sigma \eta$,

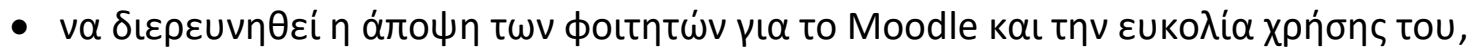

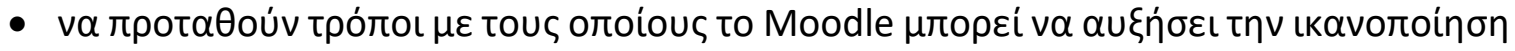

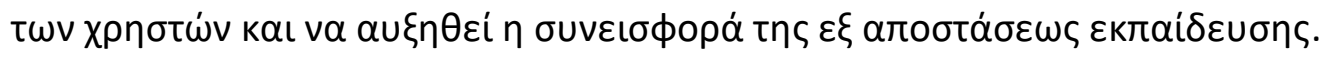

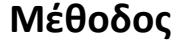

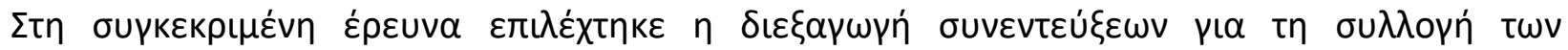

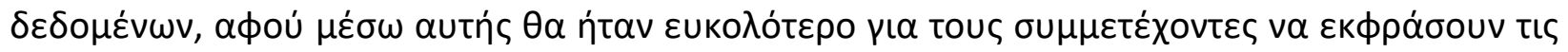

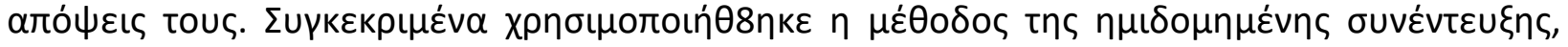

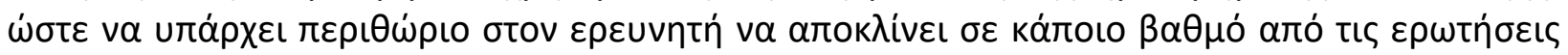

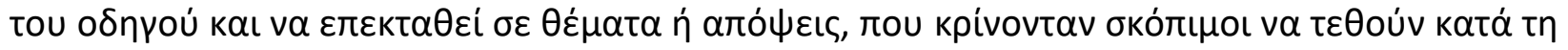

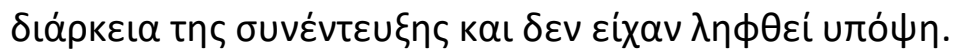

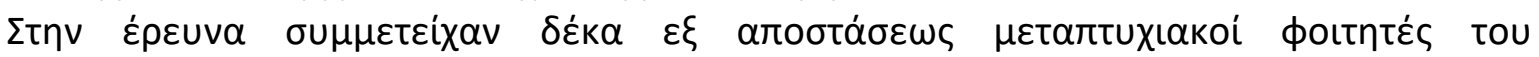

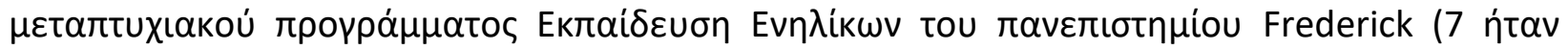

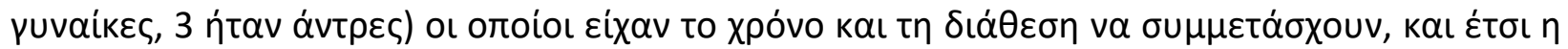

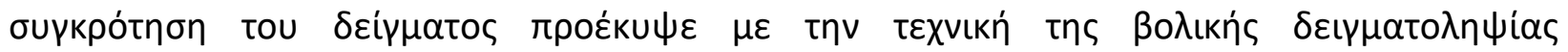

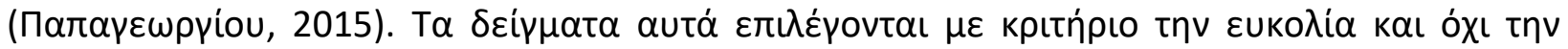

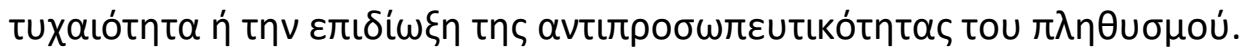

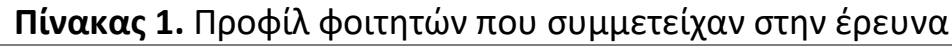

\begin{tabular}{|c|c|c|c|}
\hline 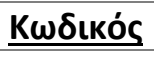 & Фúdo & 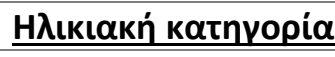 & 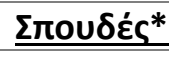 \\
\hline$\Sigma 1$ & Гuvaíka & $36-45$ & 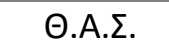 \\
\hline$\Sigma 2$ & Гuvaík $\alpha$ & $22-35$ & 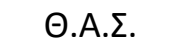 \\
\hline$\Sigma 3$ & Гuvaík $\alpha$ & $46-55$ & 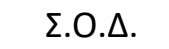 \\
\hline$\Sigma 4$ & 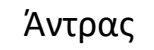 & $46-55$ & $\Sigma . П$. \\
\hline$\Sigma 5$ & Гuvaík $\alpha$ & $22-35$ & 乏.П. \\
\hline$\Sigma 6$ & 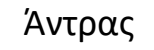 & $46-55$ & 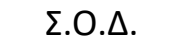 \\
\hline$\Sigma 7$ & 'Avtрas & $36-45$ & 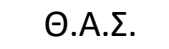 \\
\hline$\Sigma 8$ & Гuvaík $\alpha$ & $36-45$ & 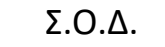 \\
\hline$\Sigma 9$ & Гuvaík $\alpha$ & $36-45$ & 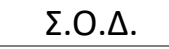 \\
\hline$\Sigma 10$ & Гuvaík $\alpha$ & $22-35$ & O.A. $\Sigma$. \\
\hline
\end{tabular}

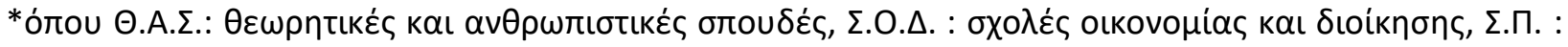

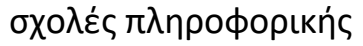


MULTILINGUAL ACADEMIC JOURNAL OF EDUCATION AND SOCIAL SCIENCES

Vol. 8 No. 1, 2020, E-ISSN: 2308-0876 @ 2020 KWP

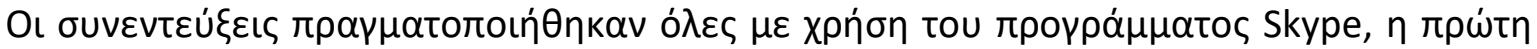

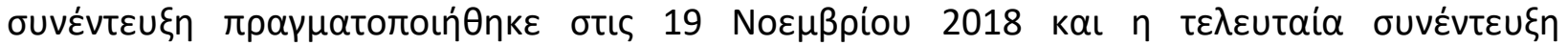

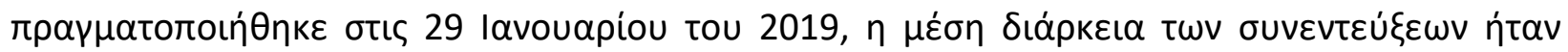

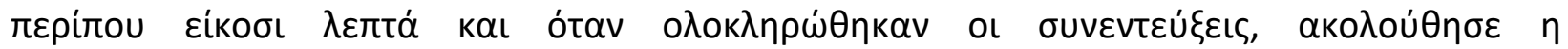

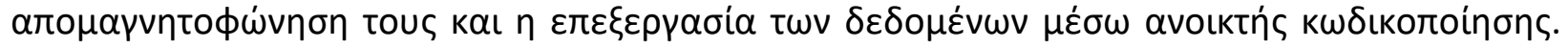

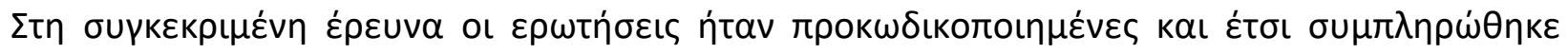

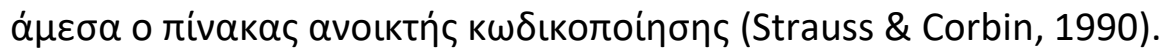

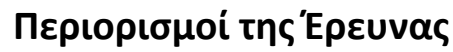

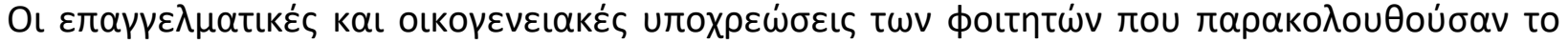

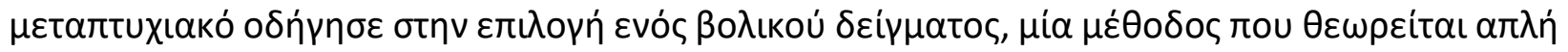

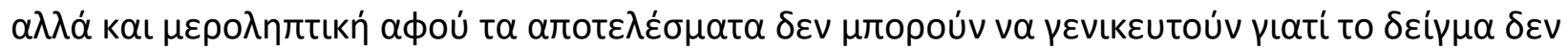

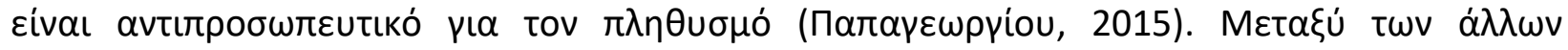

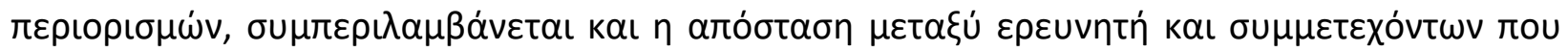

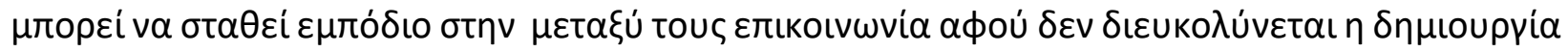

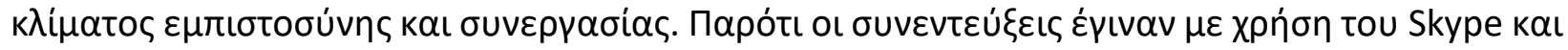

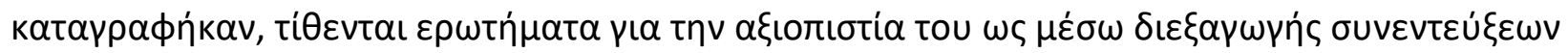

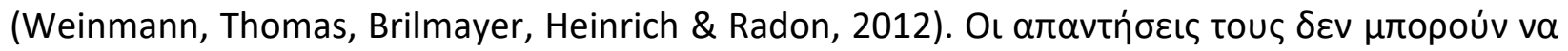

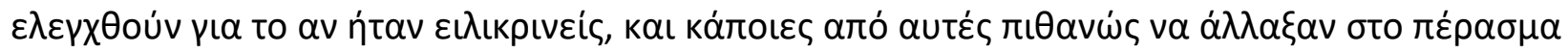
tou xpóvou.

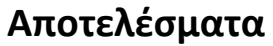

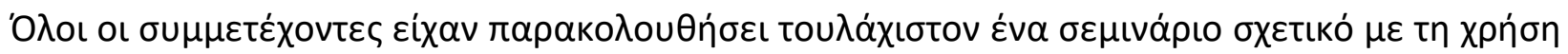

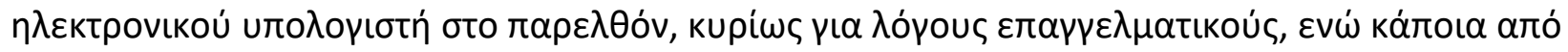

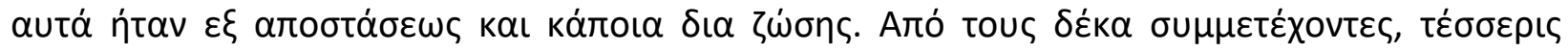

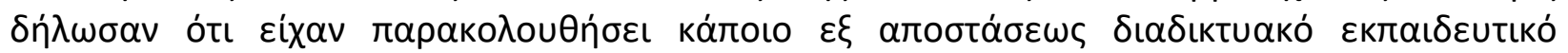

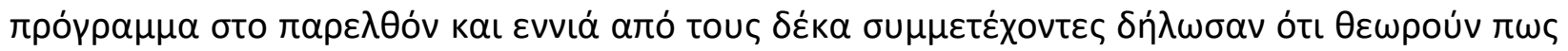

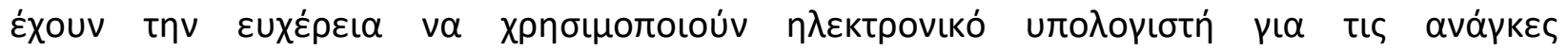

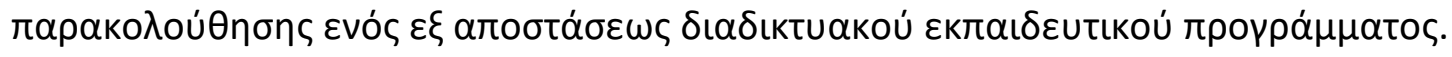

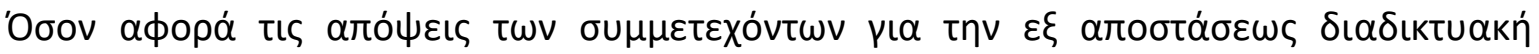

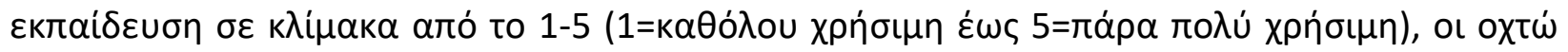

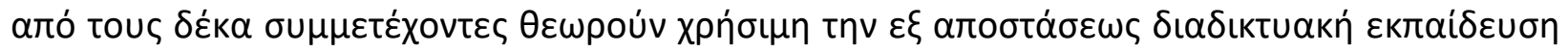

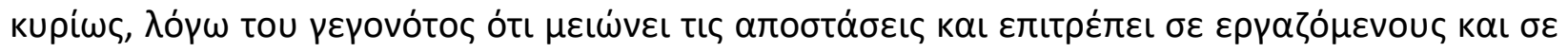

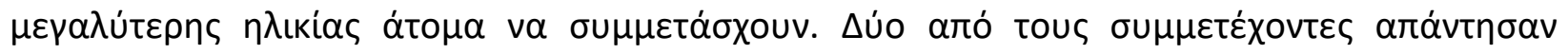

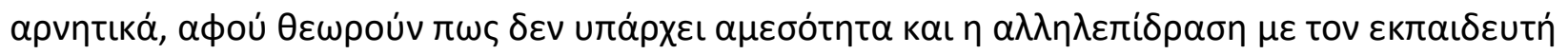

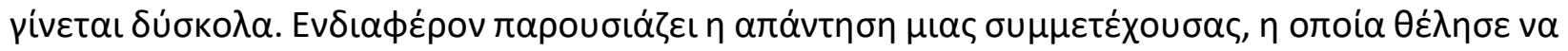

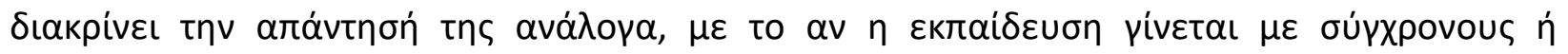

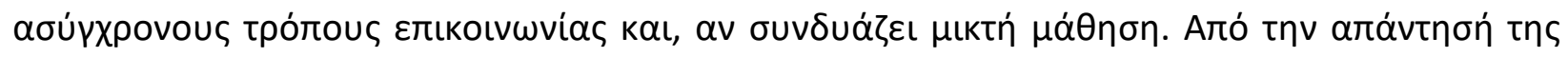

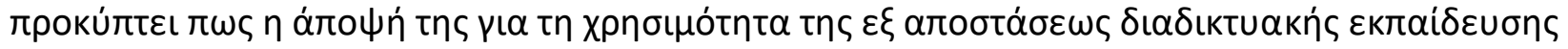

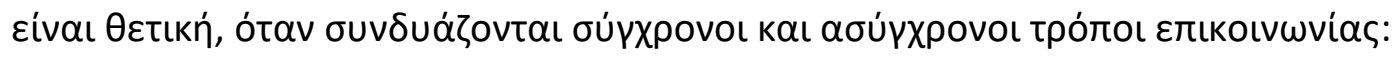

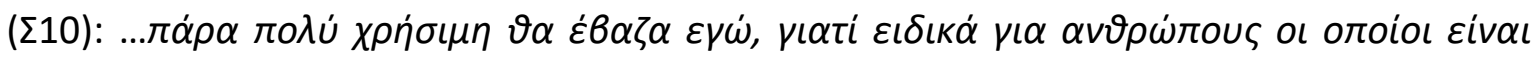

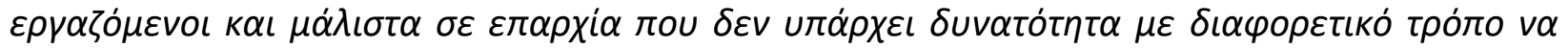

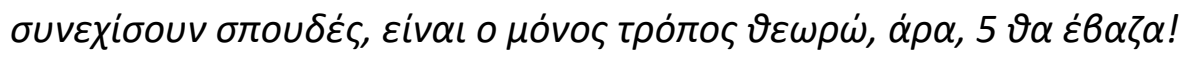


MULTILINGUAL ACADEMIC JOURNAL OF EDUCATION AND SOCIAL SCIENCES

Vol. 8 No. 1, 2020, E-ISSN: 2308-0876 @ 2020 KWP

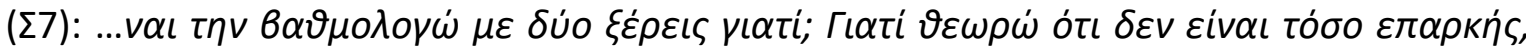

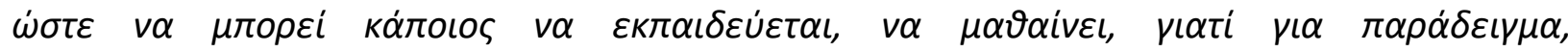

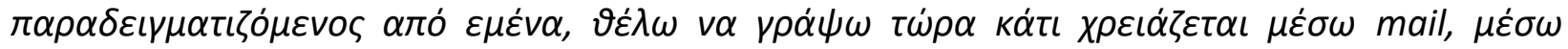

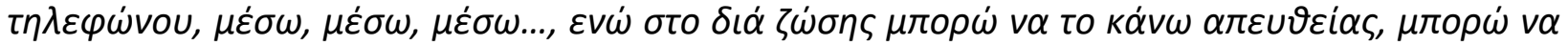

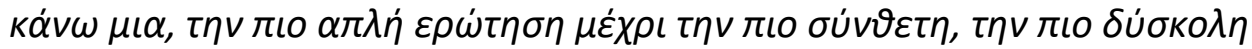

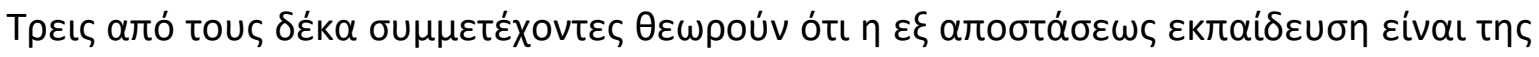

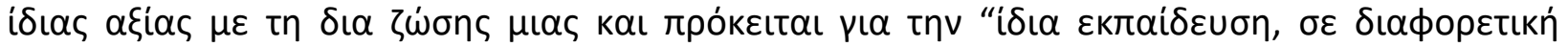

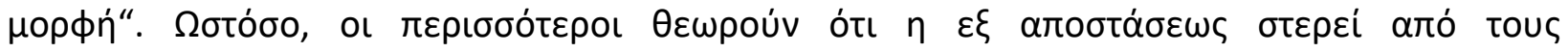

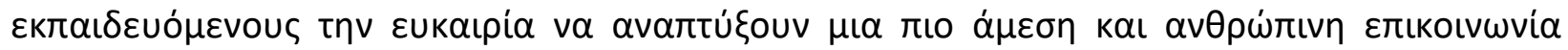
$\mu \varepsilon \tau \alpha \xi u ́$ touৎ:

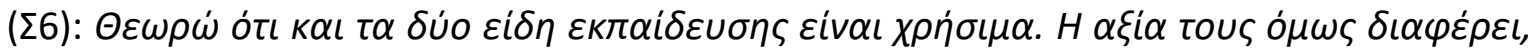

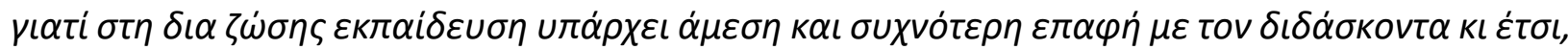

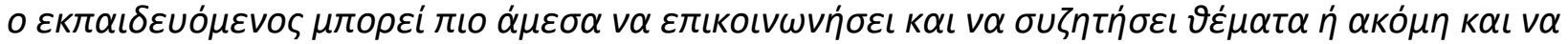

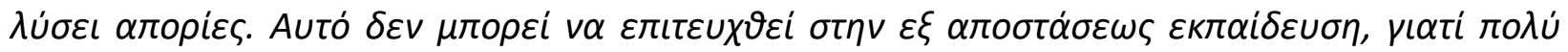

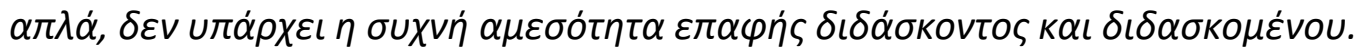

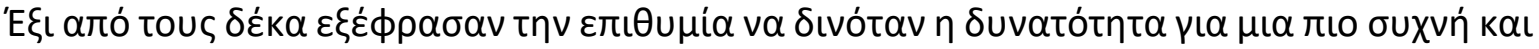

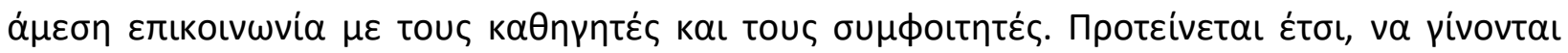

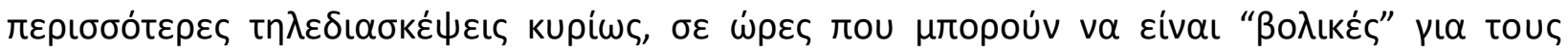

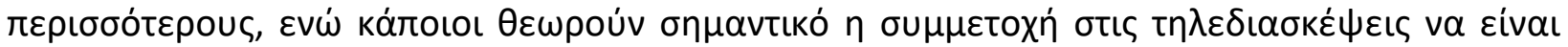

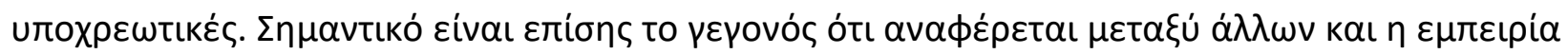

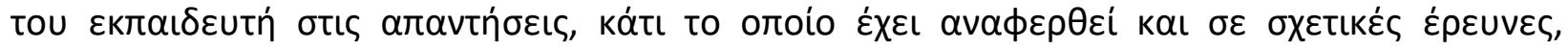

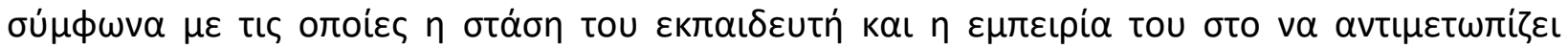

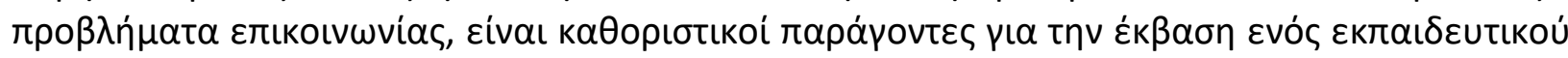

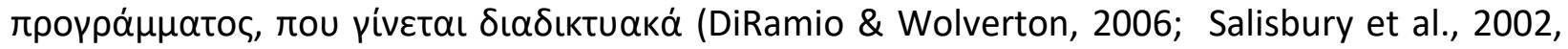

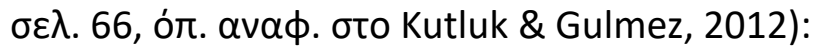

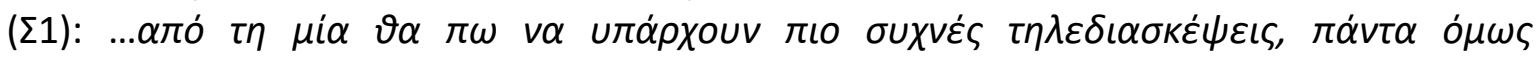

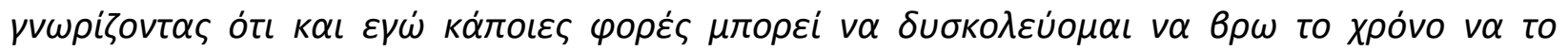

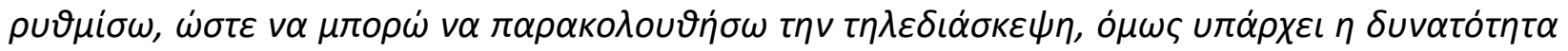

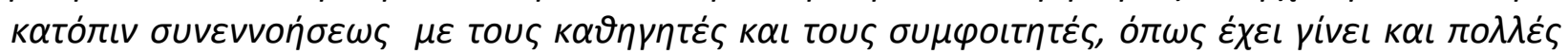

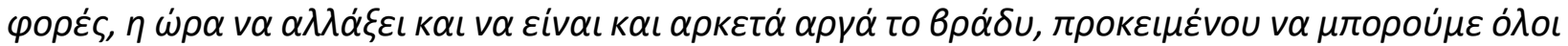

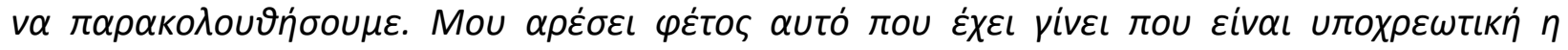

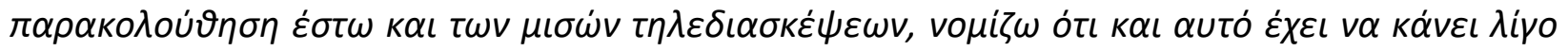

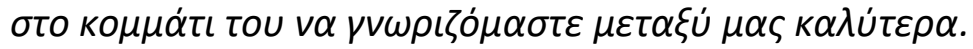

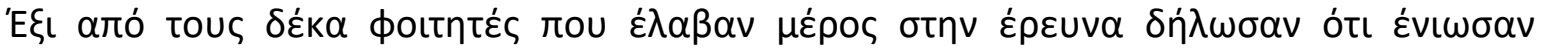

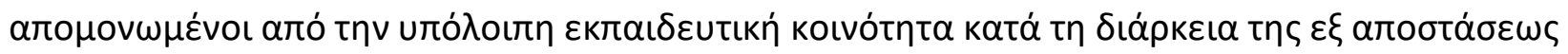

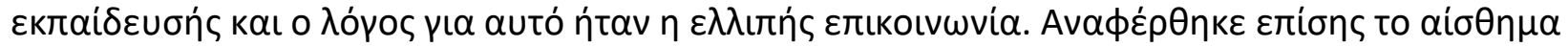

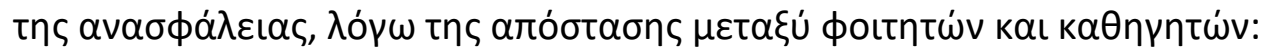

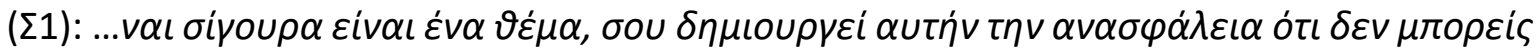

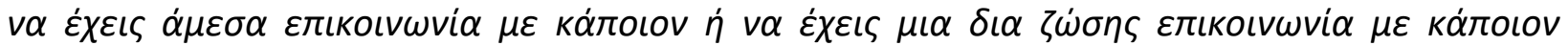

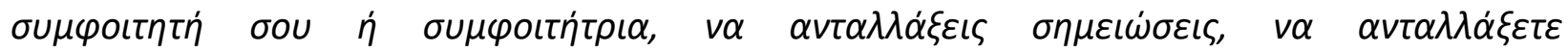

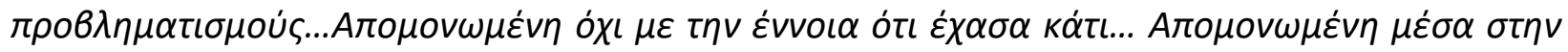

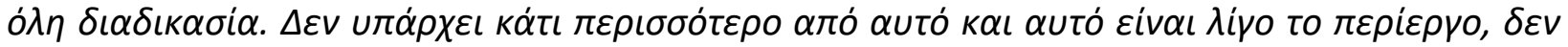

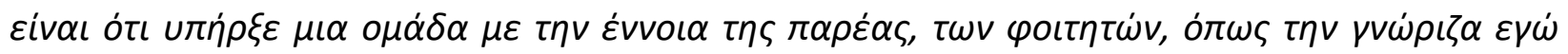


MULTILINGUAL ACADEMIC JOURNAL OF EDUCATION AND SOCIAL SCIENCES

Vol. 8 No. 1, 2020, E-ISSN: 2308-0876 @ 2020 KWP

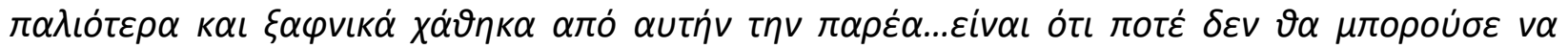

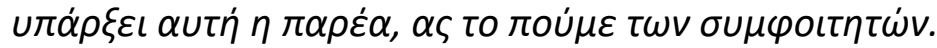

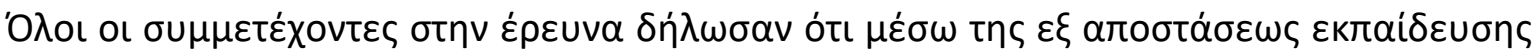

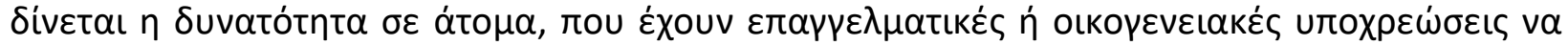

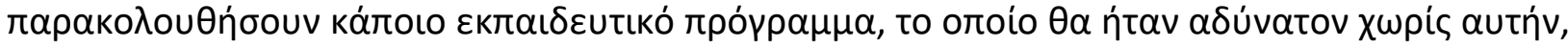

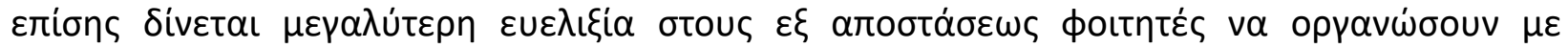

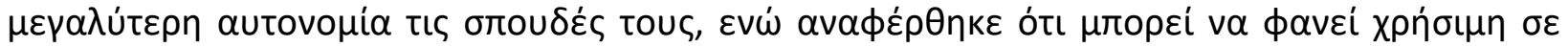

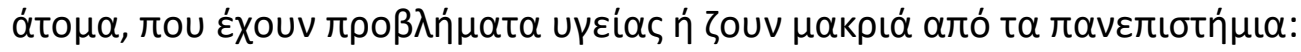

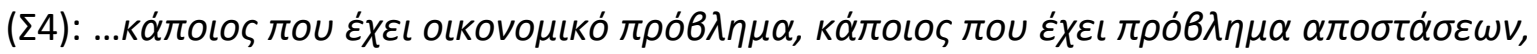

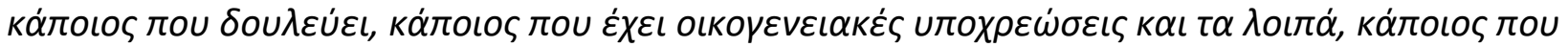

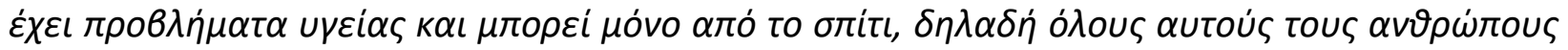

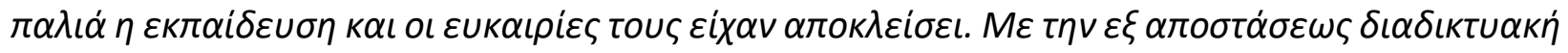

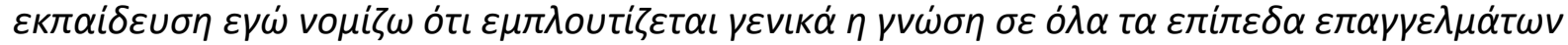

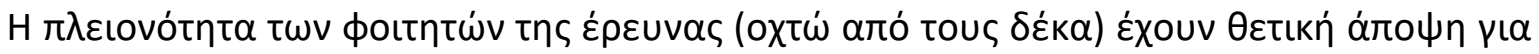

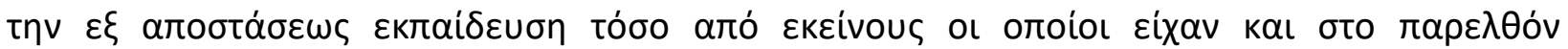

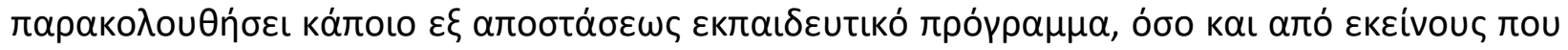

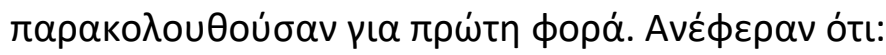

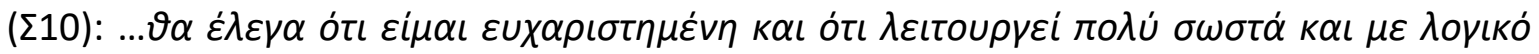

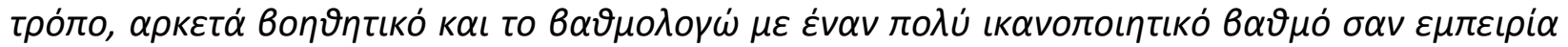

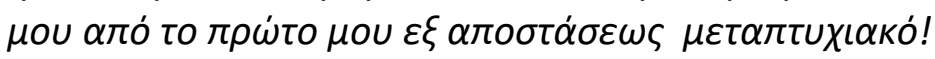

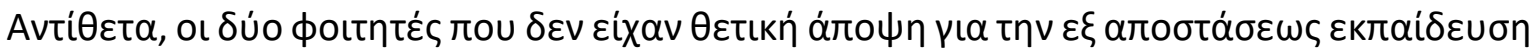

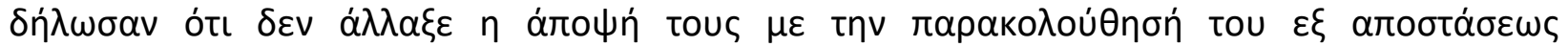

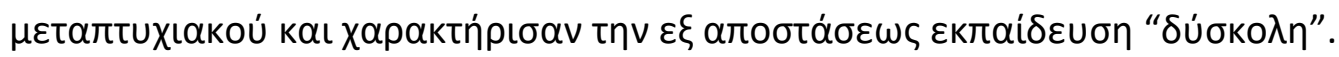

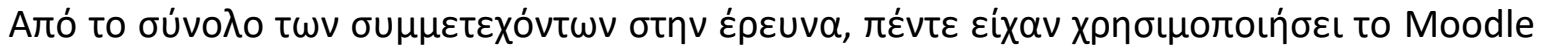

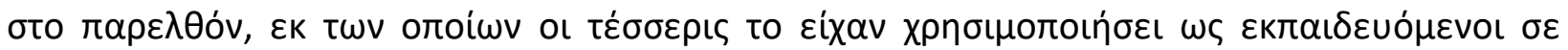

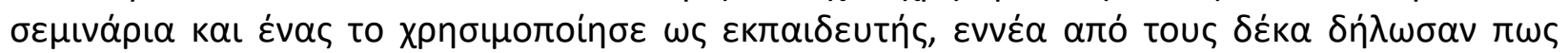

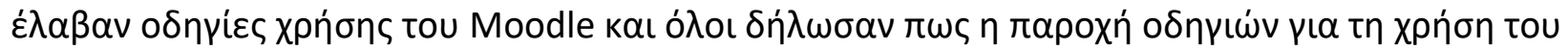

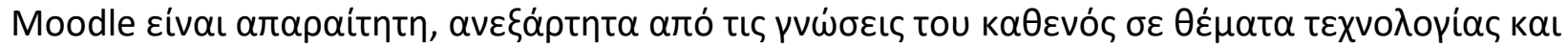

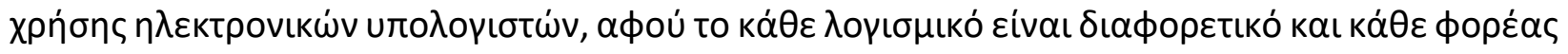

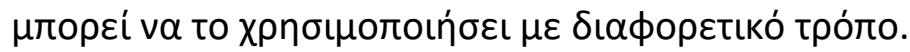

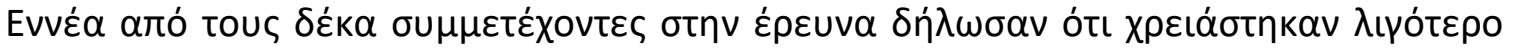

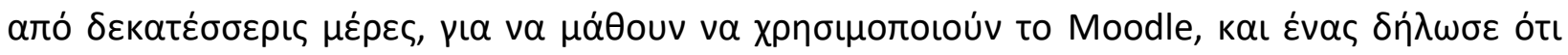

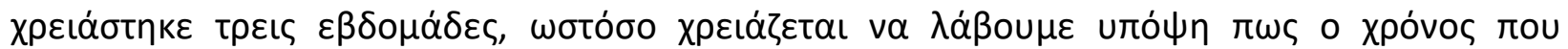

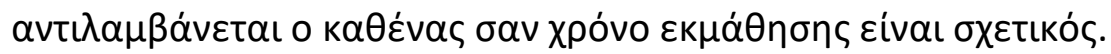

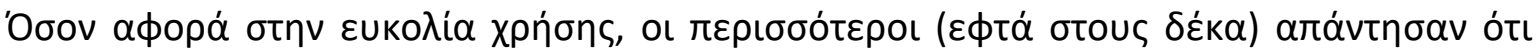

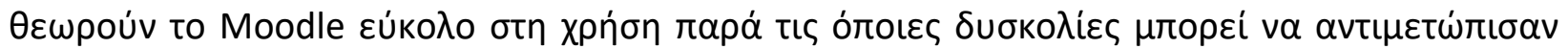

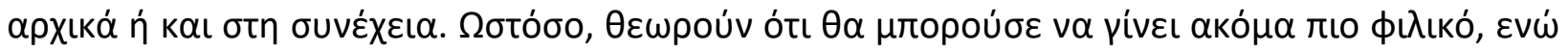

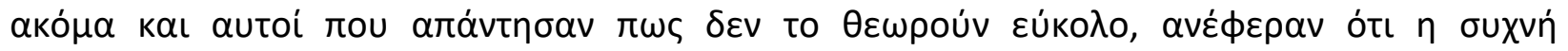

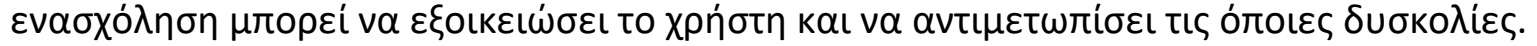

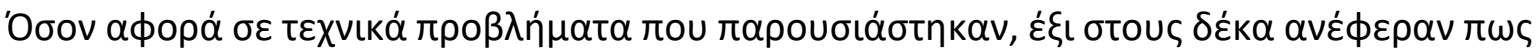

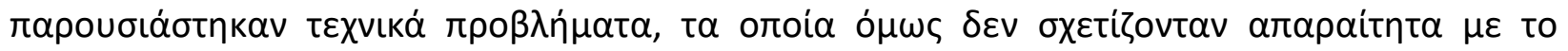

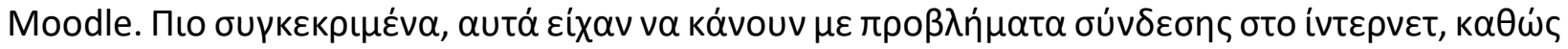

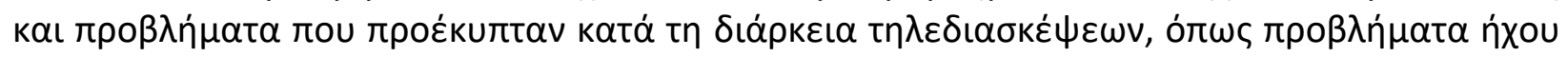

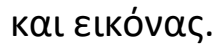


MULTILINGUAL ACADEMIC JOURNAL OF EDUCATION AND SOCIAL SCIENCES

Vol. 8 No. 1, 2020, E-ISSN: 2308-0876 @ 2020 KWP

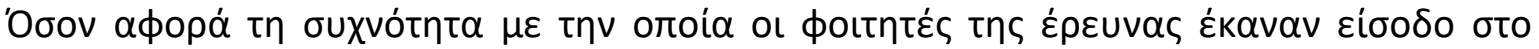

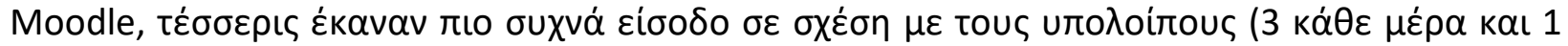

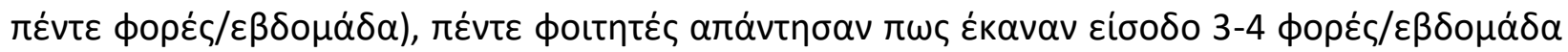

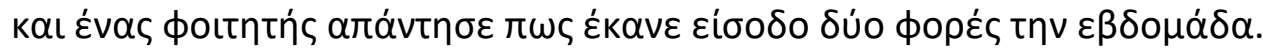

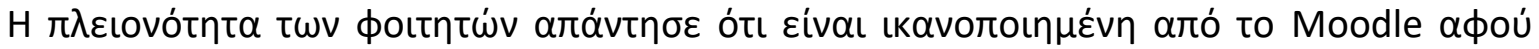

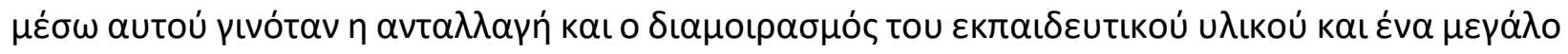

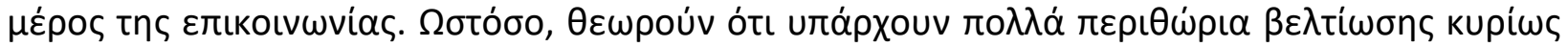

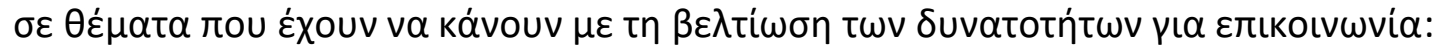

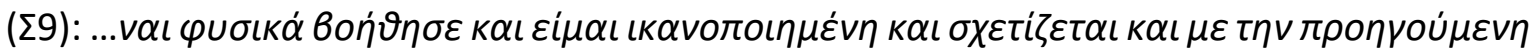

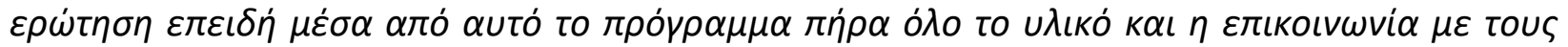

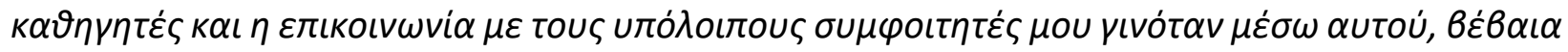

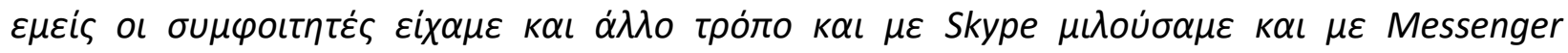

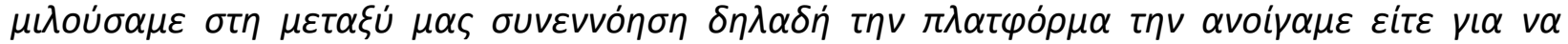

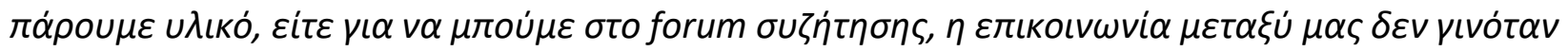

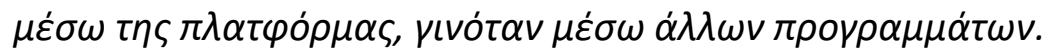

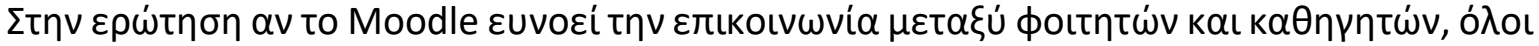

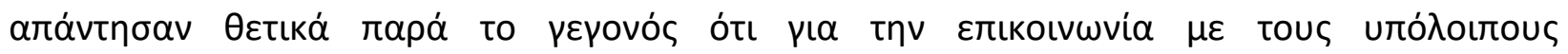

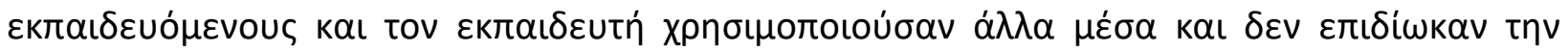

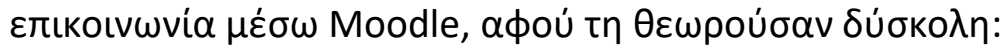

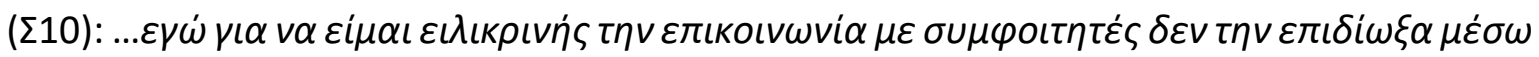

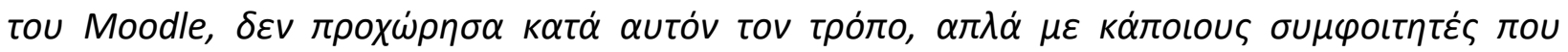

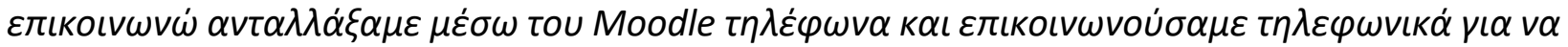

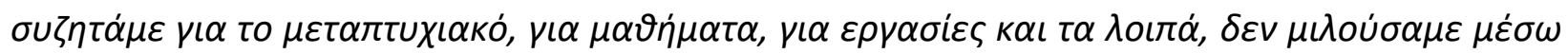

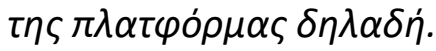

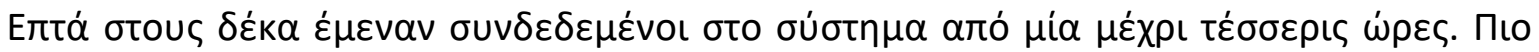

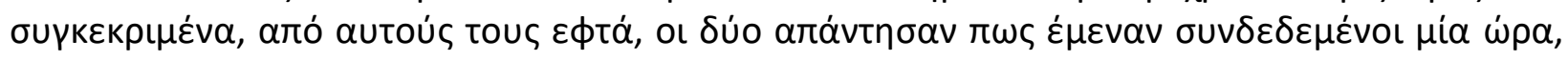

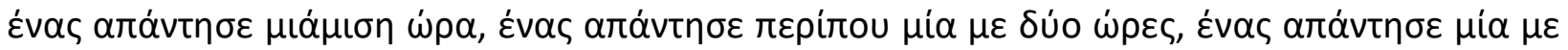

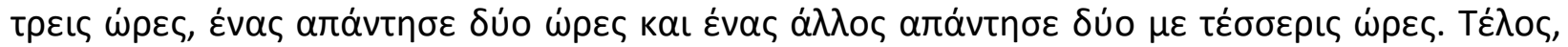

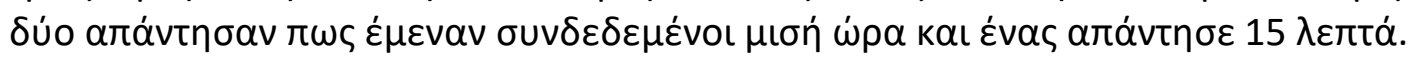

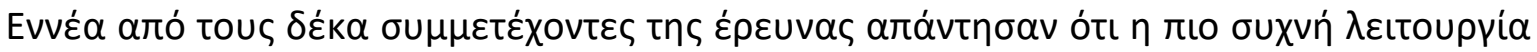

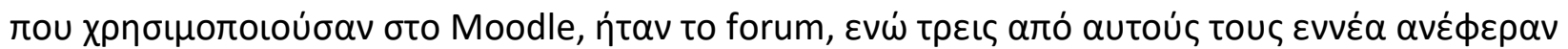

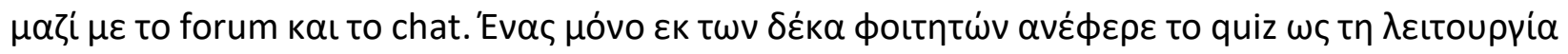

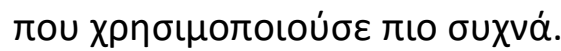

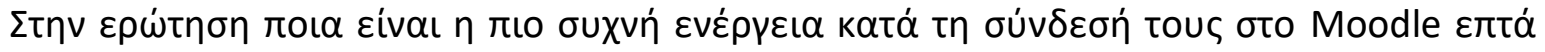

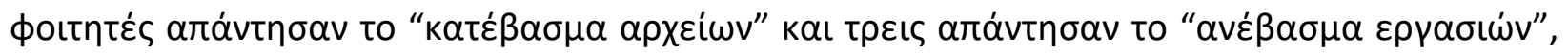

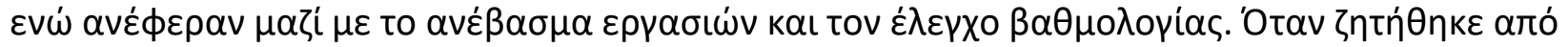

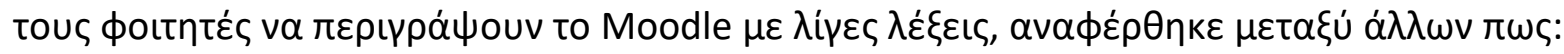

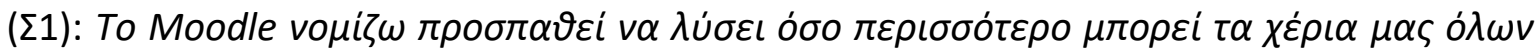

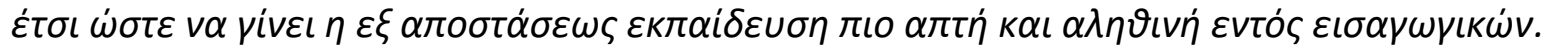

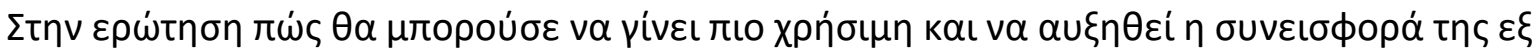

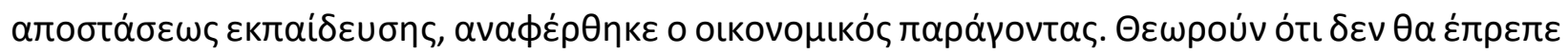

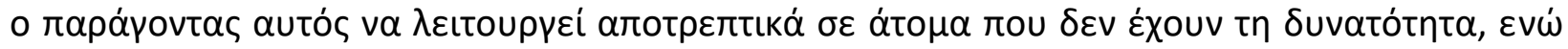

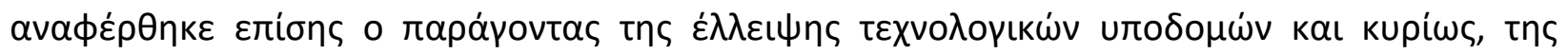

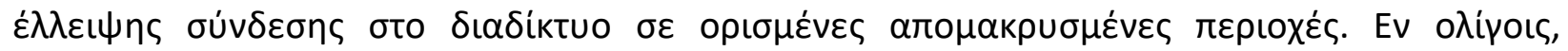


MULTILINGUAL ACADEMIC JOURNAL OF EDUCATION AND SOCIAL SCIENCES

Vol. 8 No. 1, 2020, E-ISSN: 2308-0876 @ 2020 KWP

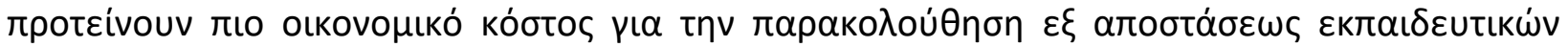

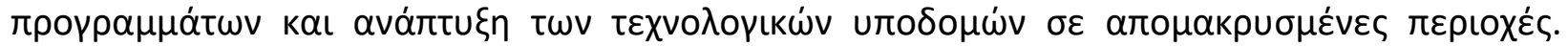

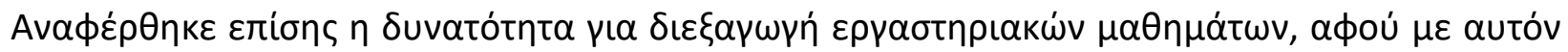

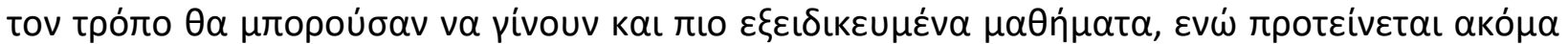

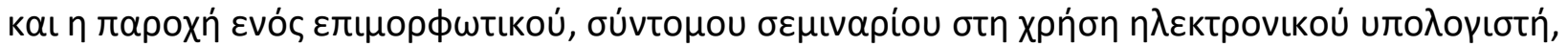

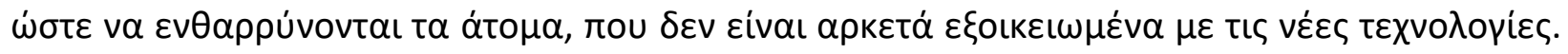

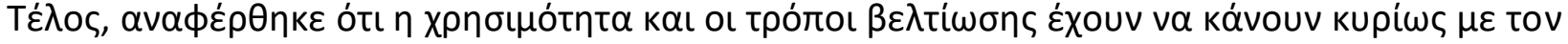

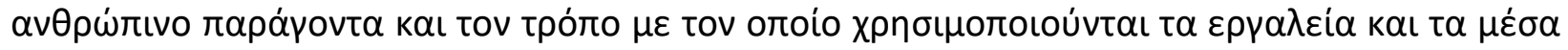

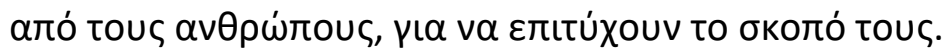

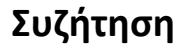

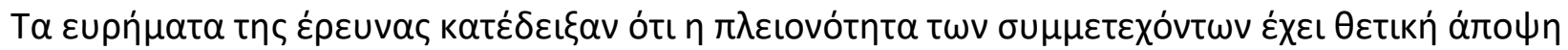

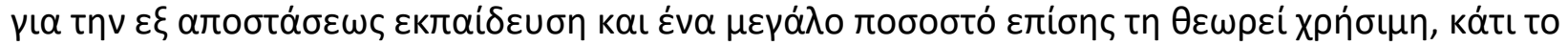

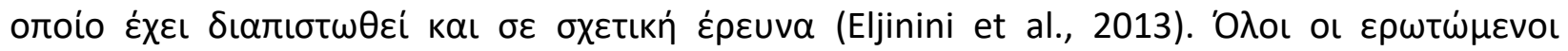

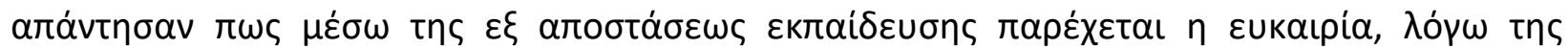

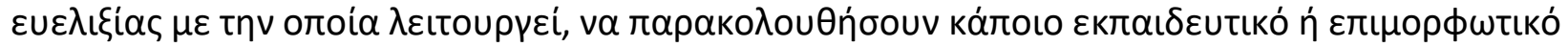

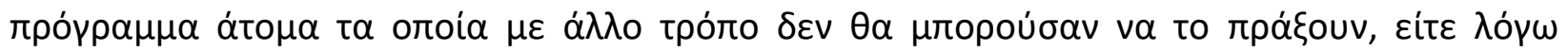

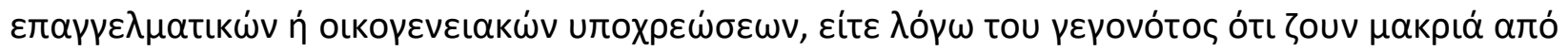

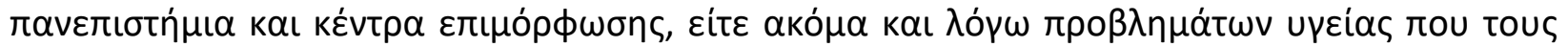

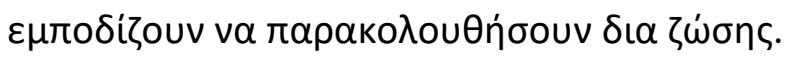

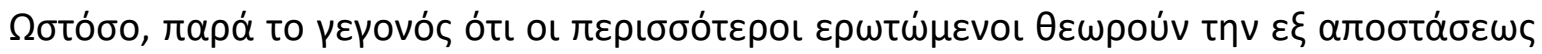

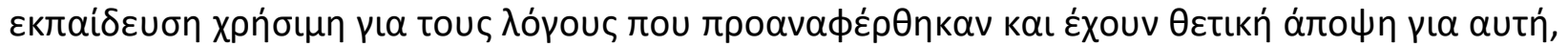

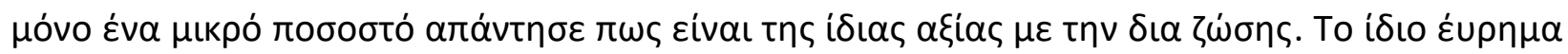

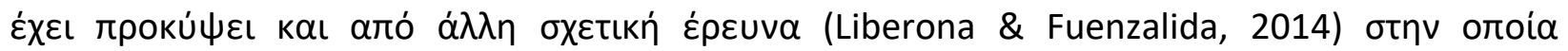

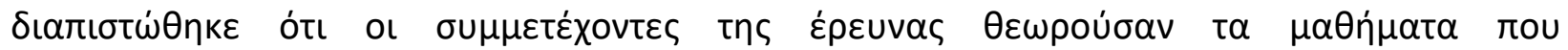

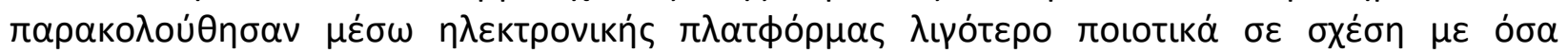

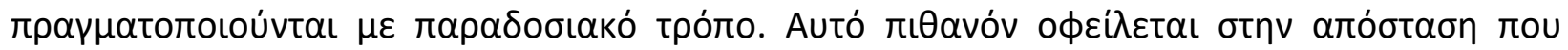

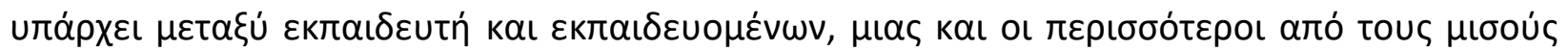

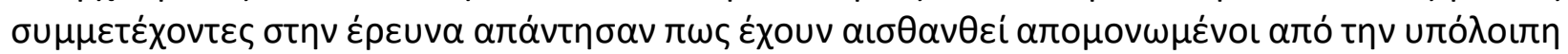

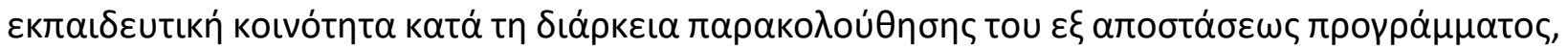

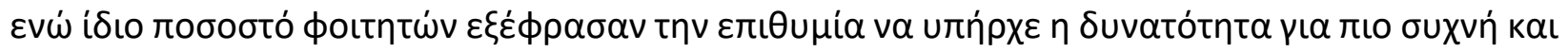

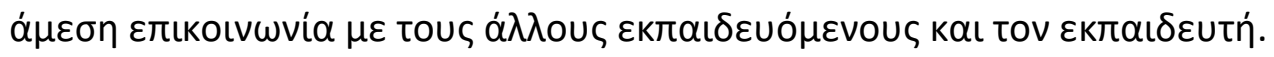

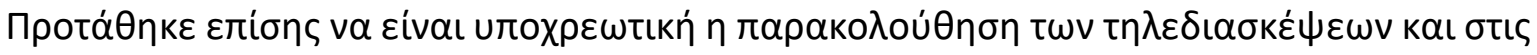

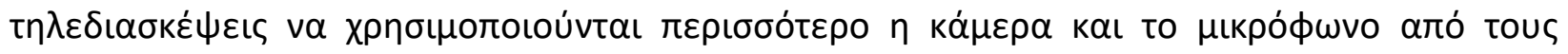

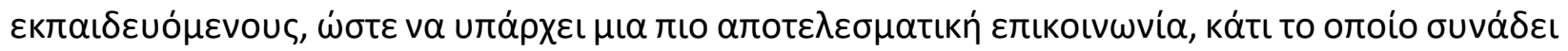

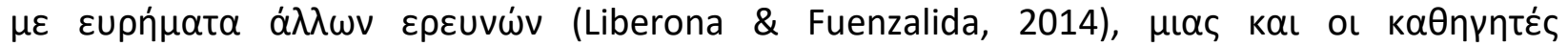

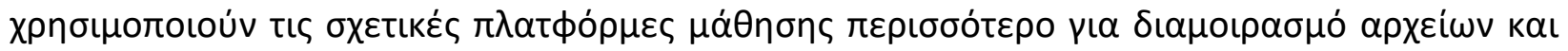

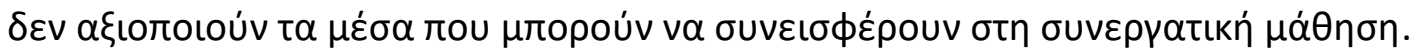

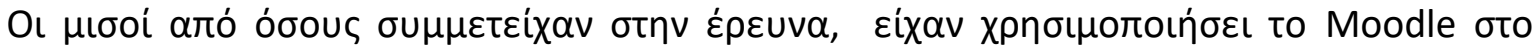

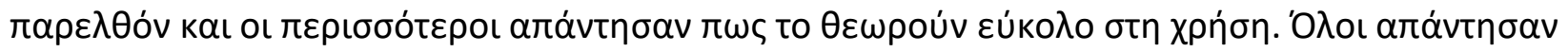

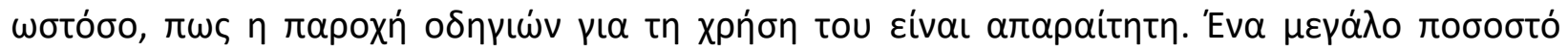

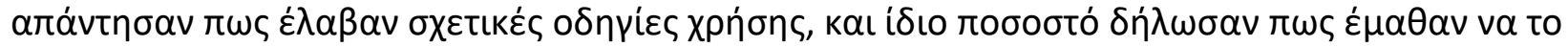

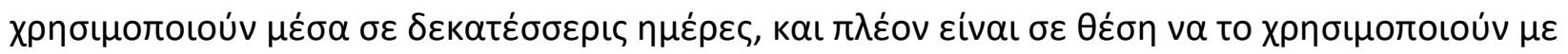

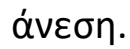


MULTILINGUAL ACADEMIC JOURNAL OF EDUCATION AND SOCIAL SCIENCES

Vol. 8 No. 1, 2020, E-ISSN: 2308-0876 @ 2020 KWP

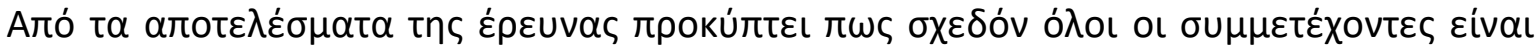

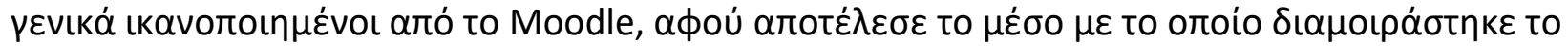

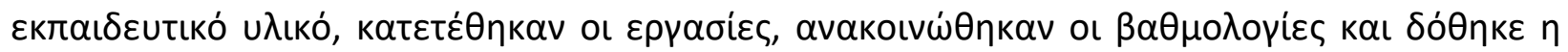

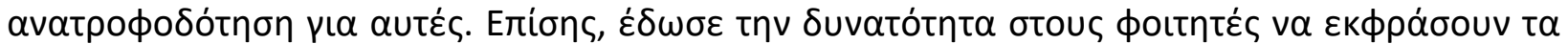

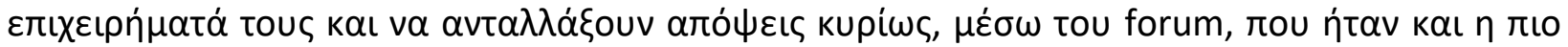

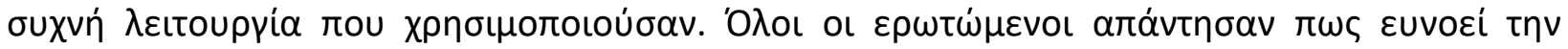

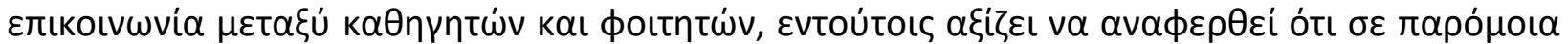

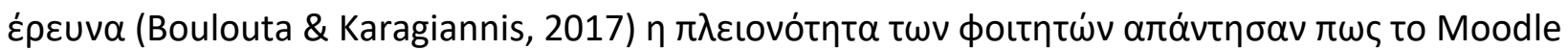

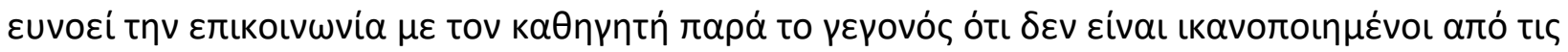

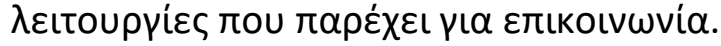

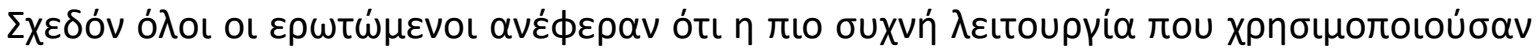

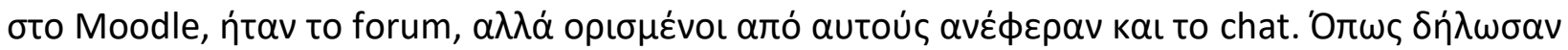

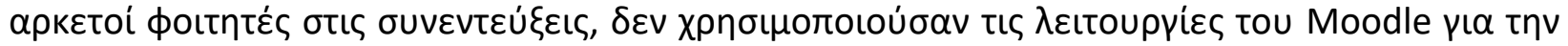

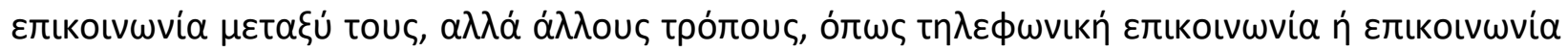

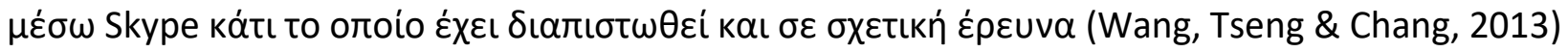

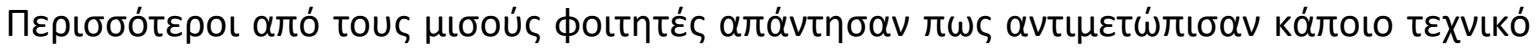

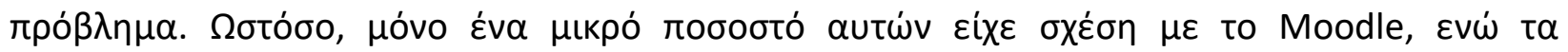

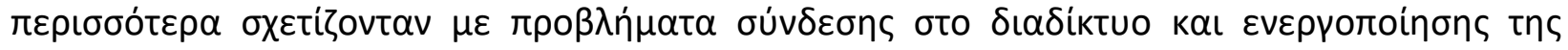

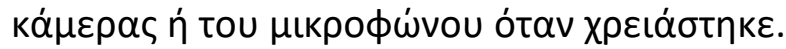

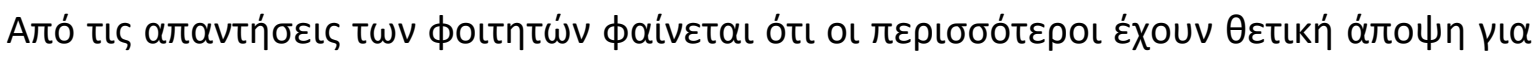

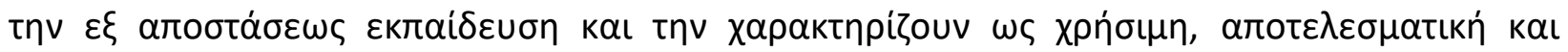

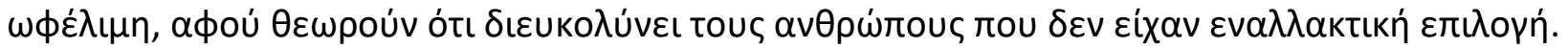

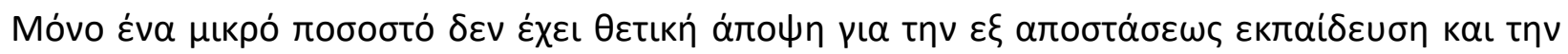

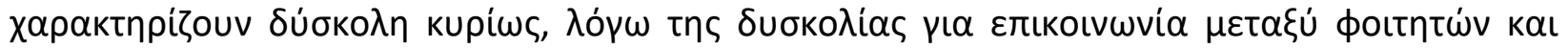

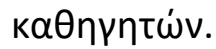

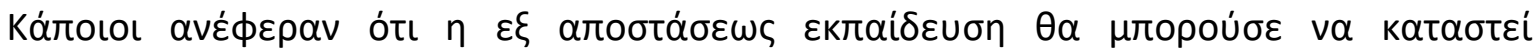

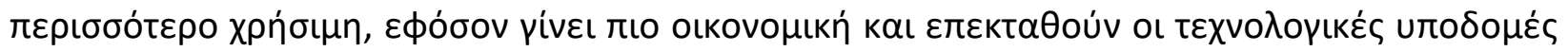

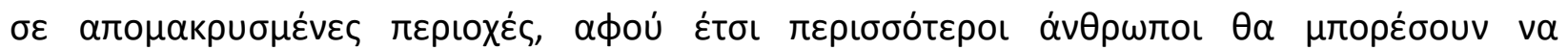

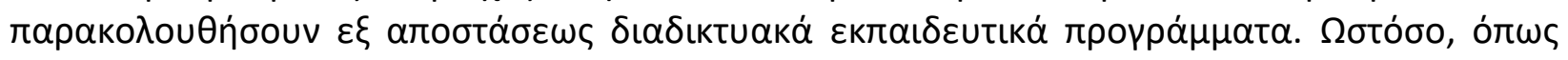

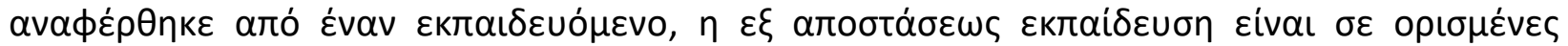

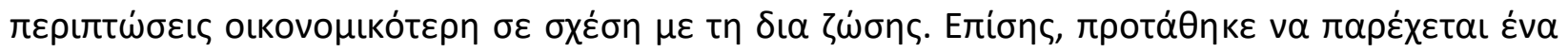

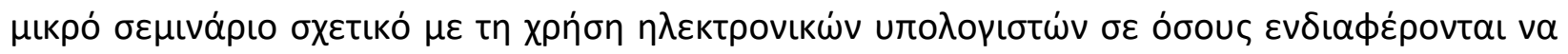

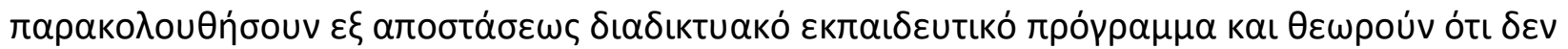

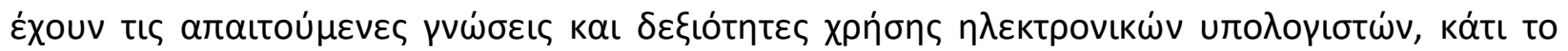

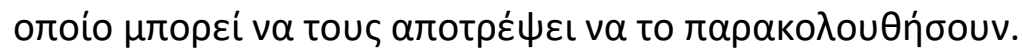

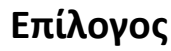

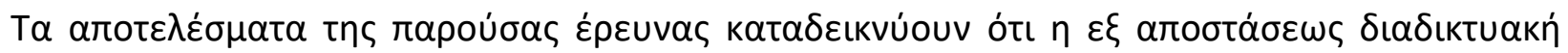

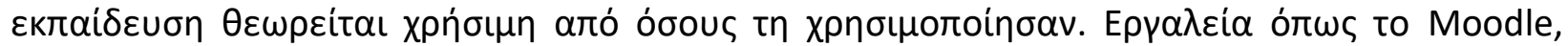

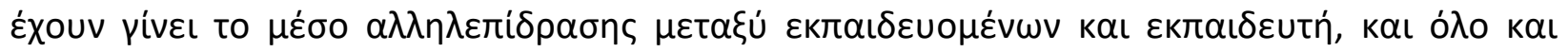

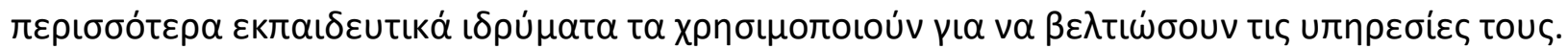

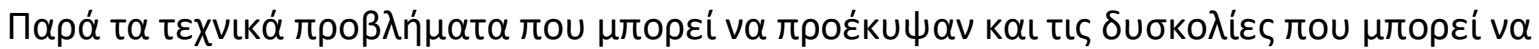

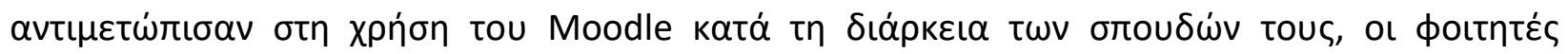


MULTILINGUAL ACADEMIC JOURNAL OF EDUCATION AND SOCIAL SCIENCES

Vol. 8 No. 1, 2020, E-ISSN: 2308-0876 @ 2020 KWP

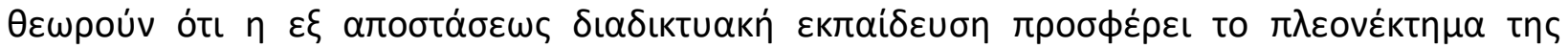

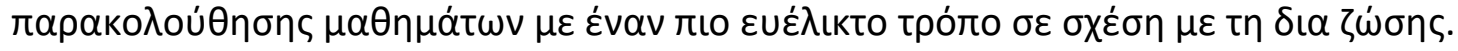

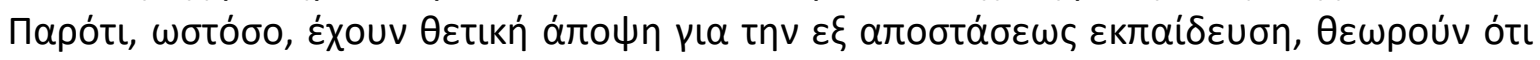

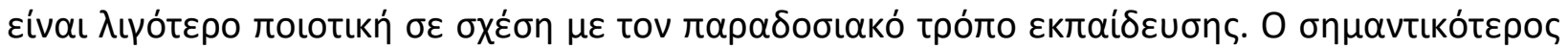

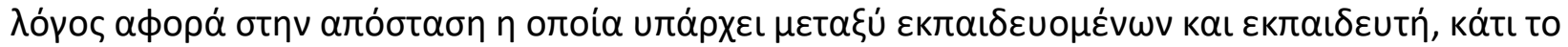

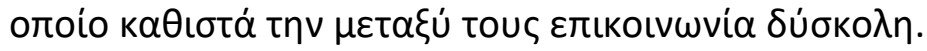

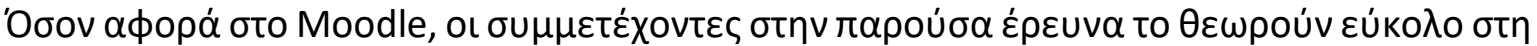

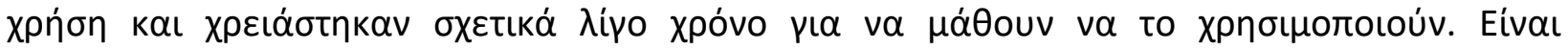

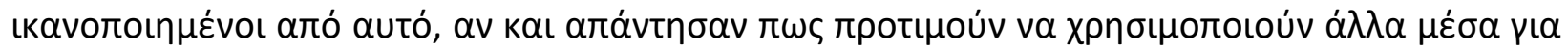

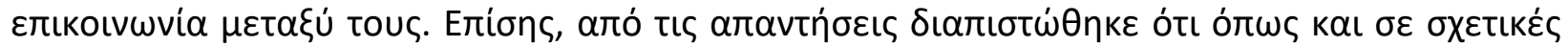

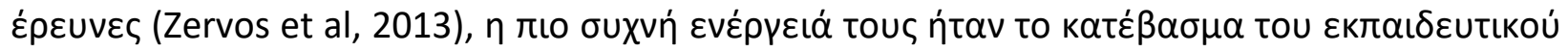

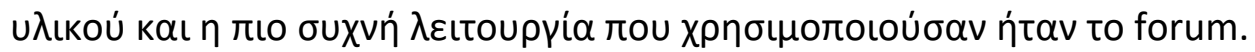

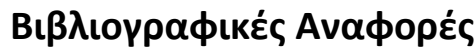 \\ References}

Al-Arimi, A. (2014). Distance Learning. Procedia - Social and Behavioral Sciences, 152, 82-88. doi: 10.1016/j.sbspro.2014.09.159.

Alzgool, M. (2019). Nexus between green HRM and green management towards fostering green values. Management Science Letters, 9(12), 2073-2082.

Arrosagaray, M., González-Peiteado, M., Pino-Juste, M., \& Rodríguez-López, B. (2019). A comparative study of Spanish adult students' attitudes to ICT in classroom, blended and distance language learning modes. Computers \& Education, 134, 31-40. doi: 10.1016/j.compedu.2019.01.016.

Boulouta, K., \& Karagiannis, S. (2017). Moodle as a Key Tool in Students Organization and Communication When Participating in Online Distance Courses. Eurasian Business Perspectives, 305-314. doi: 10.1007/978-3-319-67913-6_21.

Cardinali, F. (2006). Innovating eLearning and Mobile Learning Technologies for Europe's Future Educational Challenges, Theory and Case Studies. In W. Nejdl \& K. Tochtermann, Innovative Approaches for Learning and Knowledge Sharing (pp. 2-7). Berlin: Springer.

Cavus, N. (2015). Distance Learning and Learning Management Systems. Procedia - Social and Behavioral Sciences, 191, 872-877. doi: 10.1016/j.sbspro.2015.04.611.

Cavus, N., \& Alhih, M. (2014). Learning Management Systems Use in Science Education. Procedia - Social and Behavioral Sciences, 143, 517-520. doi: 10.1016/j.sbspro.2014.07.429.

Cobb, T. (2006). Constructivism. Encyclopedia of Language \& Linguistics, 85-87. doi: 10.1016/b008-044854-2/01593-5.

Deschacht, N., \& Goeman, K. (2015). The effect of blended learning on course persistence and performance of adult learners: A difference-in-differences analysis. Computers \& Education, 87, 83-89. doi: 10.1016/j.compedu.2015.03.020.

Detel, W. (2001). Social Constructivism. International Encyclopedia of The Social \& Behavioral Sciences, 14264-14267. doi: 10.1016/b0-08-043076-7/01086-x.

DiRamio, D., \& Wolverton, M. (2006). Integrating Learning Communities and Distance Education: Possibility or Pipedream? Innovative Higher Education, 31(2), 99-113. doi: 10.1007/s10755006-9011-y. 
MULTILINGUAL ACADEMIC JOURNAL OF EDUCATION AND SOCIAL SCIENCES

Vol. 8 No. 1, 2020, E-ISSN: 2308-0876 @ 2020 KWP

Eljinini, M., Muhsen, Z., Maaita, A., Alnsour, A., Azzam, M., \& Barhoum, K. (2013). The Utilization of the Moodle E-Learning System in Isra University. Advances in Intelligent Systems And Computing, 75-81. doi: 10.1007/978-3-319-00569-0_10.

Guri-Rosenblit, S. (2005). 'Distance education' and 'e-learning': Not the same thing. Higher Education, 49(4), 467-493. doi: 10.1007/s10734-004-0040-0.

Hlynka, D. (2013). Educational Technology. In R. Richey, Encyclopedia of Terminology for Educational Communications and Technology (pp. 102-104). Springer.

Khalid, N., Islam, D. M. Z., \& Ahmed, M. R. M. (2019). SENTREPRENEURIAL TRAINING AND ORGANIZATIONAL PERFORMANCE: IMPLICATIONS FOR FUTURE. Humanities \& Social Sciences Reviews, 7(2), 590-593.

Kor, H., Aksoy, H., \& Erbay, H. (2014). Comparison of the Proficiency Level of the Course Materials (Animations, Videos, Simulations, E-books) Used in Distance Education. Procedia - Social and Behavioral Sciences, 141, 854-860. doi: 10.1016/j.sbspro.2014.05.150.

Kossieris, P., Panayiotakis, A., Tzouka, K., Gerakopoulou, P., Rozos, E., \& Makropoulos, C. (2014). An eLearning Approach for Improving Household Water Efficiency. Procedia Engineering, 89, 1113-1119. doi: 10.1016/j.proeng.2014.11.232.

Kutluk, F., \& Gulmez, M. (2012). A Research about Distance Education Students' Satisfaction with Education Quality at an Accounting Program. Procedia - Social and Behavioral Sciences, 46, 2733-2737. doi: 10.1016/j.sbspro.2012.05.556.

Liberona, D., \& Fuenzalida, D. (2014). Use of Moodle Platforms in Higher Education: A Chilean Case. In L. Uden, J. Sinclair, Y. Tao \& D. Liberona, Learning Technology for Education in Cloud (pp. 124-134). Springer.

Lock, J. (2013). Educational Technology. In R. Richey, Encyclopedia of Terminology for Educational Communications and Technology (pp. 95-96). Springer.

Manea, A. (2014). Lifelong Learning Programs-An Effective Means of Supporting Continuing Education. Procedia - Social and Behavioral Sciences, 142, 454-458. doi: 10.1016/j.sbspro.2014.07.648.

Moodle.org: Moodle Statistics. (2018). Retrieved September 28, 2018, from https://moodle.net/stats/.

Oliver, A., Tomás, J., \& Montoro-Rodriguez, J. (2017). Dispositional hope and life satisfaction among older adults attending lifelong learning programs. Archives of Gerontology and Geriatrics, 72, 80-85. doi: 10.1016/j.archger.2017.05.008.

Strauss, A., \& Corbin, J. (1990). Basics of Qualitative Research: Grounded Theory procedures and techniques. Sage: Newbury Park.

Thang, L., Lim, E., \& Tan, S. (2018). Lifelong learning and productive aging among the babyboomers in Singapore. Social Science \& Medicine. doi: 10.1016/j.socscimed.2018.08.021.

Trif, L. (2015). Training Models of Social Constructivism. Teaching Based on Developing A Scaffold. Procedia - Social and Behavioral Sciences, 180, 978-983. doi: 10.1016/j.sbspro.2015.02.184.

Tudor, S. (2013). Formal - Non-formal - Informal in Education. Procedia - Social and Behavioral Sciences, 76, 821-826. doi: 10.1016/j.sbspro.2013.04.213.

Umrani, W., Ahmed, U., \& Memon, P. (2015). Examining the absorptive capacity construct: A validation study in the Pakistani banking context. Management Science Letters, 5(12), 1053-1058. 
Wang, Y., Tseng, Y., \& Chang, C. (2013). Comparison of Students' Perception of Moodle in a Taiwan University against Students in a Portuguese University. In J. Wang \& R. Lau, Advances in Web-Based Learning - ICWL 2013 (pp. 71-78). Berlin: Springer.

Weinmann, T., Thomas, S., Brilmayer, S., Heinrich, S., \& Radon, K. (2012). Testing Skype as an interview method in epidemiologic research: response and feasibility. International Journal of Public Health, 57(6), 959-961. doi: 10.1007/s00038-012-0404-7.

Zervos, S., Kyriaki-Manessi, D., Koulouris, A., Giannakopoulos, G., \& Kouis, D. (2013). Evaluation of the e-Class Platform of the LIS Dept., TEI of Athens. Procedia - Social and Behavioral Sciences, 73, 727-735. doi: 10.1016/j.sbspro.2013.02.111.

Zin, M. L. M., \& Ibrahim, H. (2020). The Influence of Entrepreneurial Supports on Business Performance among Rural Entrepreneurs. Annals of Contemporary Developments in Management \& HR (ACDMHR), 2(1), 31-41.

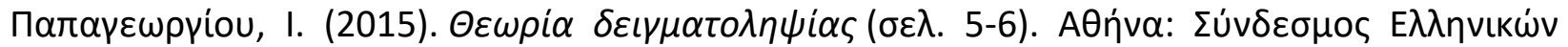

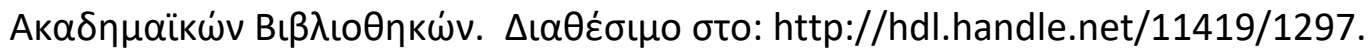

